\title{
Smart Energy and Spectral Efficiency (SE) of Distribution Broadband over Power Lines (BPL) Networks - Part 2: L1PMA, L2WPMA and L2CXCV for SE against Measurement Differences in Overhead Medium-Voltage BPL Networks
}

\author{
Athanasios G. Lazaropoulos* \\ School of Electrical and Computer Engineering / National Technical University of Athens / \\ 9 Iroon Polytechniou Street / Zografou, GR 15780
}

Received June 7, 2018; Accepted August 17, 2018; Published August 21, 2018

\begin{abstract}
This second paper assesses the performance of piecewise monotonic data approximations, such as L1PMA, L2WPMA and L2CXCV, against the measurement differences during the spectral efficiency (SE) calculations in overhead medium-voltage broadband over power lines (OV MV BPL) networks. In this case study paper, the performance of the aforementioned three already known piecewise monotonic data approximations, which are considered as countermeasure techniques against measurement differences, is here extended during the SE computations. The indicative BPL topologies of the first paper are again considered while the $3-30 \mathrm{MHz}$ frequency band of the $\mathrm{BPL}$ operation is assumed.
\end{abstract}

Keywords: Smart Grid; Broadband over Power Lines (BPL); Power Line Communications (PLC); Distribution Power Grid; Spectral Efficiency.

\section{Introduction}

Smart energy is a sustainable and worthwhile energy system where energy production, transmission and delivery are integrated and coordinated with the energy consumption, smart grid applications, energy services, active producers / consumers, renewable / storage solutions and enabling communications technologies. However, the emerging advanced IP-based power network requires high spectral efficiency (SE) potential so that the supported plethora of relevant broadband applications can be easily supported [1]-[3].

Among the enabling communications technology solutions of the smart grid, Broadband over Power Lines (BPL) technology attracts the interest of many decision makers due to the low installation cost of the BPL devices on the existing power grid infrastructure. Already presented in [4], the spectral behavior of distribution BPL networks -i.e., overhead (OV) and underground (UN) medium-voltage (MV) and low-voltage (LV) BPL networks- is assessed through the hybrid model [5]-[22]. In this paper, CS2 module, which constitutes a refinement of the hybrid model concerning its containing top-down approach, is adopted. On the basis of the SE, 
the impact of CS2 module and of the measurement differences between the experimental and theoretical results on the broadband performance of the distribution BPL networks has been assessed in [4]. Here, it should be noted that measurement differences have been handled as error distributions and especially by following continuous uniform distributions (CUDs) of maximum and minimum value $a_{\mathrm{CUD}}$ and $-a_{\mathrm{CUD}}$, respectively. The assessment has been accomplished through the lens of appropriate statistical performance metrics of SE that are classified into two metric sets. Set A consists of the maximum, minimum and average SE while Set B comprises the mean absolute error (MAE) and the root mean square deviation (RMSD) of SE.

To mitigate the aforementioned measurement differences that further affect the statistical performance metrics, three well-known piecewise monotonic data approximations (i.e., L1PMA, L2WPMA and L2CXCV) are going to be applied [23]-[32]. Until now, L1PMA, L2WPMA and L2CXCV have been applied and examined in transmission and distribution BPL networks in order to counteract the occurred measurement differences during the channel attenuation computations [31]-[37]. Useful results, which are going to be adopted in this paper, concerning the application properties of piecewise monotonic data approximations against the measurement differences during the channel attenuation computations have been deduced in [36], [37]. On the basis of these application properties, the application of the piecewise monotonic data approximations is extended to the SE results in order to reveal the theoretical SE values by ignoring the fluctuations of channel attenuation computations due to the measurement differences and without knowing the CUD measurement difference properties (i.e., maximum and minimum values of the CUD measurement distributions). The countermeasure efficiency of the L1PMA, L2WPMA and L2CXCV against the measurement differences during the SE computation is assessed for comparison reasons on the basis of: (i) the main performance metric of the piecewise monotonic data approximation method [33], [36], that is the percent error sum (PES); and (ii) the statistical performance metrics of set A and B that already been applied in [4].

The rest of this paper is organized as follows: In Sec.2, a brief presentation of the L1PMA, L2WPMA and L2CXCV is given. Sec.3 synopsizes the applied performance metrics, already been presented in [4]. The strong and the weak points of the performance metrics are demonstrated. Sec. 4 discusses the simulation results of various distribution BPL topologies intending to mark out the efficiency of L1PMA, L2WPMA and L2CXCV against the restoration of the theoretical SE when measurement differences of various CUD properties are considered. Sec.5 concludes this paper.

\section{Measurement Differences and Piecewise Monotonic Data Approximation Methods}

\subsection{Measured and Theoretical SE}

As already been mentioned in [4], [36], [37], a number of practical reasons and "real-life" conditions create significant differences between experimental measurements and theoretical results during the determination of transfer functions. However, these transfer function computation fluctuations affect SE.

In fact, the measurement differences that occur during the transfer function determination of BPL networks indirectly affect the determination of SE. Numerically, 
when measurement differences are taken into consideration, the measured distribution BPL coupling transfer function $\overline{H^{\mathrm{C}}}\{\cdot\}$ for given coupling scheme $C$ is determined by

$$
\overline{H^{\mathrm{C}}}\left(f_{i}\right)=H^{\mathrm{C}}\left(f_{i}\right)+e\left(f_{i}\right)
$$

where $f_{i}, i=1, \ldots, u$ denotes the measurement frequency, $H^{C}\left(f_{i}\right)$ is the theoretical coupling transfer function that is given by the application of the hybrid model presented in [4], $e\left(f_{i}\right)$ synopsizes the total measurement difference that follows continuous uniform distributions (CUDs) in $\mathrm{dB}$ with minimum value $-a_{\mathrm{CUD}}$ and maximum value $a_{\mathrm{CUD}}$ and $u$ is the number of subchannels in the examined frequency range. On the basis of eq. (3) of [4], the measured and theoretical SE for given coupling scheme channel $C$ can be respectively determined by

$$
\begin{aligned}
& \overline{S E^{C}}\left(f_{i}\right)=\log _{2}\left\{1+\left[\frac{\left\langle p\left(f_{i}\right)\right\rangle_{L}}{\left\langle N\left(f_{i}\right)\right\rangle_{L}} \cdot\left|\overline{H^{\mathrm{C}}}\left(f_{i}\right)\right|^{2}\right]\right\}, i=1, \ldots, u \\
& S E^{C}\left(f_{i}\right)=\log _{2}\left\{1+\left[\frac{\left\langle p\left(f_{i}\right)\right\rangle_{L}}{\left\langle N\left(f_{i}\right)\right\rangle_{L}} \cdot\left|H^{C}\left(f_{i}\right)\right|^{2}\right]\right\}, i=1, \ldots, u
\end{aligned}
$$

where $p\{\cdot\}$ are appropriate IPSD limits expressed in $\mathrm{dBm} / \mathrm{Hz}$ that ensure the low electromagnetic interference (EMI) of BPL system operation to the other telecommunication systems that operate at the same frequency band, $N\{\cdot\}$ are uniform additive white Gaussian noise (AWGN) PSD levels expressed in $\mathrm{dBm} / \mathrm{Hz}$ and $\langle\cdot\rangle_{L}$ is an operator that converts $\mathrm{dBm} / \mathrm{Hz}$ into a linear power ratio $(\mathrm{W} / \mathrm{Hz})$. By comparing eqs. (2) and (3), it is evident that SE depends on the assumed coupling transfer function while significant differences between measured and theoretical coupling transfer functions entail differences between measured and theoretical SE.

In order to cope with the measurement differences, various monotonic data approximation methods, which treated as countermeasure techniques against measurement differences, have been proposed by Demetriou, such as L1PMA, L2WPMA and L2CXCV [23]-[32]. Their countermeasure efficiency against measurement differences during the determination of the coupling transfer functions of distribution BPL networks has been extensively validated in [34]-[41] while a number of useful smart grid applications concerning the power grid monitoring and control have been proposed on the basis of these piecewise monotonic data approximations. Here, the aforementioned three piecewise monotonic data approximations are applied in order to reveal the theoretical SE when measured SE, the distribution BPL topology and the applied coupling scheme are already known. Actually, piecewise monotonic data approximations aim at mitigating the deviations between the measured and the theoretical SE which are the result of the existence of measurement differences. With reference to eq. (2) and (3), piecewise monotonic data approximations give as output the approximated SE for given coupling scheme channel $C$, say:

$$
\overline{\overline{S E^{C}}}\left(f_{i}\right)=q\left\{\overline{S E^{C}}\left(f_{i}\right)\right\}, i=1, \ldots, u
$$

where $q\{\cdot\}$ is the general function of the applied piecewise monotonic data approximations. It should be noted that during the determination of the approximated SE of eq. (4), CUD properties of measurement differences are not known.

To apply the aforementioned three piecewise monotonic data approximations (i.e., L1PMA, L2WPMA and L2CXCV) and to determine the piecewise monotonic data approximation of eq. (4), the special application characteristics of each one are mentioned in the following subsections.

\subsection{L1PMA}


L1PMA decomposes the input data (i.e., SE data in this paper) into separate monotonous sections between its adjacent turning points (primary extrema) by exploiting the piecewise monotonicity property of transmission and distribution BPL transfer functions [25], [26]. On the basis of the minimization of the moduli sum between the output data (L1PMA approximation data of SE) and input data into the separate monotonous sections, L1PMA achieves to mitigate the uncorrelated SE differences, which come from the assumed measurement differences, by neglecting the existence of few large ones [33], [36], [37]. The L1PMA application is based on the Fortran software package that is freely available online in [42] receives as inputs the measured SE, the measurement frequencies and the number of monotonic sections (i.e., either user- or computer-defined) and primarily gives as output the best fit of the measured SE. As already been presented in [36], [37], critical role during the computation of the best of the measured SE plays the selection of the number of the monotonic sections.

\subsection{L2WPMA}

Similarly to L1PMA, L2WPMA decomposes the examined input data (i.e., either transfer function data or SE data of this paper), which are contaminated by measurement differences, into separate monotonous sections between its primary extrema [31], [36], [37]. L2WPMA is implemented by a Fortran software package that is freely available online in [31]. As in L1PMA case, L2WPMA software receives as input the measured $\mathrm{SE}$, the measurement frequencies and the number of monotonic sections and primarily gives as output a spline representation of the measured SE. Conversely to L1PMA, L2WPMA focuses on the first divided of input data and demands the minimization of the weighted sum of the square of the measurement differences by requiring specific number of sign changes. The number of sign changes is equal to the number of monotonic sections minus one. Again, the number of monotonic sections is either user- or computer-defined.

\subsection{L2CXCV}

In accordance with [32], [36], [37], L2CXCV smooths the input data with measurement differences in the least square error sense. In contrast with L1PMA and L2WPMA, L2CXCV does not focus on the number of monotonic sections but on the second divided differences of the smoothed values by solving a strictly convex quadratic programming problem for each set [32]. Similarly to L1PMA and L2CXCV, the Fortran software package that is applied to implement L2CXCV is freely available online in [43]. In general, $\mathrm{L} 2 \mathrm{CXCV}$ receives as input the measured $\mathrm{SE}$ and gives as output the fit of the measured SE.

\section{Performance Metrics}

\subsection{PES}

In accordance with [33], [36], PES is the main performance metric that is employed to assess the approximation accuracy when piecewise monotonic data approximation methods are applied in BPL networks. More specifically, in this paper, PES expresses as a percentage the total sum of the relative differences between the examined SE and the theoretical SE for all the used frequencies. There are two submetrics of PES that should be compared in order to benchmark the mitigation efficiency of the applied piecewise monotonic data approximation method, say: 
- PES $S_{\text {meas: }}$ This PES submetric examines the relative difference between the SE, which is affected by the measurement differences, and the theoretical SE. With reference to eqs. (2) and (3), $P E S_{\text {meas }}$ is given by:

$$
P E S_{\text {meas }}=100 \% \cdot \frac{\sum_{i=1}^{u}\left|\overline{S E^{C}}\left(f_{i}\right)-S E^{C}\left(f_{i}\right)\right|}{\sum_{i=1}^{u}\left|S E^{C}\left(f_{i}\right)\right|}
$$

- $P E S_{\text {approx: }}$ This PES submetric examines the relative difference between the approximated and the theoretical SE. With reference to eqs. (3) and (4), $P E S_{\text {approx }}$ is given by:

$$
P E S_{\text {approx }}=100 \% \cdot \frac{\sum_{i=1}^{u}\left|\overline{\overline{S E C}}\left(f_{i}\right)-S E^{C}\left(f_{i}\right)\right|}{\sum_{i=1}^{u}\left|S E^{C}\left(f_{i}\right)\right|}
$$

From eqs. (5) and (6), the PES difference that is given by

$$
\triangle P E S=P E S_{\text {meas }}-P E S_{\text {approx }}
$$

determines the quality of the approximation. If $\triangle \mathrm{PES}$ is positive, the examined approximation method successfully mitigates the measurement differences. $\triangle \mathrm{PES}$ helps towards the determination of the optimal number of monotonic sections of L1PMA and L2WPMA [36], [37].

\subsection{Metrics of Set A and B}

In accordance with [4], two sets of performance metrics (i.e., Set A and B) can be applied in order to assess either the SE impact of measurement differences or the SE mitigation efficacy of piecewise monotonic data approximations against measurement differences. Both performance metrics sets can give benchmark results to the aforementioned two issues. In [4], both sets have been applied and have assessed the SE impact of measurement differences. On the basis of the results of [4], these sets are here adopted in order to assess the mitigation efficiency of measurement differences by three piecewise monotonic data approximations. The first set of performance metrics, which is denoted as Set A, concerns the influence of the measurement differences on the general properties of the SE results while the second set, which is denoted as Set B, assesses the SE impact intensity of measurement differences. More specifically:

- Set A: With reference to eqs. (2) and (3), it is assumed that the measured and theoretical SE for given coupling scheme channel $C$ are already known. Set A consists of the metrics of: (i) average value of SE; (ii) maximum value of $\mathrm{SE}$; and (iii) minimum value of SE. Since measured SE is infected by unbiased measurement differences, it is expected that average, maximum and minimum values remain almost the same with the respective metrics of the theoretical SE. This has already been verified in [4]. Hence, the metrics of set A are unable to identify the existence and the intensity of measurement differences although even small divergences of the three metrics indicate the existence of measurement differences. If measurement differences occur and piecewise monotonic data approximations are applied in order to mitigate these measurement differences, the set A metrics of the approximated SE of eq. (4) should remain almost the same with the respective metrics of the theoretical SE. Therefore, the set A metrics act as an integrity metric for the countermeasures techniques of [4].

- Set B: Set B consists of two metrics, say: (i) MAE; and (ii) RMSD. With reference to [4] and in contrast with the metrics of Set A, the metrics of Set B can not only detect the existence but also to assess the intensity of the 
measurement differences. In fact, the values of the Set B metrics increase with the increase of the measurement difference $a_{\text {CUD }}$. Anyway, the set $\mathrm{B}$ metrics constitute comparison metrics between measured and theoretical SE. If measurement differences occur and piecewise monotonic data approximations are applied in order to mitigate these measurement differences, the set B metrics of the approximated SE of eq. (4) should give closer values to the theoretical ones in comparison with the respective metrics of the measured SE.

\section{Numerical Results and Discussion}

Various topologies of OV MV BPL networks, which have been presented in Sec.2.2 of [4] and are also common in [36], [37], are here simulated with the purpose of comparatively benchmarking the SE mitigation efficiency of L1PMA, L2WPMA and L2CXCV against measurement differences added during the transfer function determination.

As the propagation and transmission specifications are regarded, those are the same with [33]-[37]. Arbitrarily, the $\mathrm{WtG}^{1}$ coupling scheme is applied during the following simulations. As it is usually done [5], [11], [13], [44], the selection of representative coupling schemes is a typical procedure for the sake of reducing manuscript size.

As the spectral exploitation properties are concerned, injected power spectral density limits (IPSD limits) of Ofcom are adopted [4]-[13], [45]-[51], while a uniform AWGN PSD levels $N(f)$ will be assumed equal to $-105 \mathrm{dBm} / \mathrm{Hz}$ in the case of OV MV BPL networks. In order to compute SE of OV MV BPL topologies, the BPL frequency range and flat-fading subchannel frequency spacing are first assumed equal to $3-30 \mathrm{MHz}$ and $0.1 \mathrm{MHz}$, respectively, so that the required $\mathrm{SE}$ accuracy can be preserved. However, in order to apply the piecewise monotonic data approximations, the flat-fading subchannel frequency spacing is assumed equal to $0.1 \mathrm{MHz}$. The latter specification has been made so that: (i) the results of SE can clearly be presented in the following figure; and (ii) the results concerning the selection of monotonic sections can be comparable with the respective results of [36], [37] that deal with the application behavior of piecewise monotonic data approximations in channel attenuation results. For the latter case, note that the BPL frequency range and flat-fading subchannel frequency spacing are assumed equal to $1-30 \mathrm{MHz}$ and $1 \mathrm{MHz}$, respectively, in [36], [37].

Finally, as the nature of measurement differences and the mitigation of measurement differences are concerned, unbiased measurement differences are assumed, which affect the measurements of channel attenuation -see eqs. (1) and (2) - and [4]. Measurement differences follow continuous uniform distributions (CUDs) with minimum value $-a_{\mathrm{CUD}}$ and maximum value $a_{\mathrm{CUD}}$. The piecewise monotonic data approximations are applied when the measured SE of eq. (1) are known for given maximum value $a_{\text {CUD }}$ and the approximation results of piecewise monotonic data approximations are directly compared against the theoretical SE of eq. (2). Afterwards, the aforementioned difference is compared against the difference between the measured and the theoretical SE. The mitigation efficiency of piecewise monotonic data approximations depends on the relation between the aforementioned two differences. 


\subsection{SE $\triangle P E S$ of L1PMA, L2WPMA and L2CXCV against Measurement Differences}

By simply maintaining the monotonicity pattern, piecewise monotonic data approximations achieve to mitigate the additive measurement differences so that approximations that resemble the theoretical data can be made [36]. To examine the impact of measurement differences on the SE of OV MV BPL topologies and the potential of counterbalancing the measurement differences, in Figs. 1(a)-(d), the theoretical and the measured SE are plotted versus frequency for the four indicative OV MV BPL topologies, respectively. Note that the measured SE corresponds to measurement difference CUD of $\alpha_{\mathrm{CUD}}=5 \mathrm{~dB}$. Also, in each figure, apart from the theoretical and measured SE curves, three L1PMA SE approximation curves are also presented when the number of monotonic sections is assumed to be equal to 2, 5 and 20 . In Figs. 2(a)-(d) and 3(a)-(d), same plots are given with Figs. 1(a)-(d) but for the application of L2WPMA and L2CXCV, respectively.

Comparing Figs. 1-3 and Figs. 2-4 of [36], certain similarities and differences between SE and channel attenuation approximations can be pointed out:

- In [36], piecewise monotonic data approximations have mitigated the measurement differences that had been added to the measured channel attenuation while, in this paper, piecewise monotonic data approximations try to mitigate the measured SE whose calculations are based on the measured channel attenuation. Hence, piecewise monotonic data approximations have directly mitigated the measurement differences in [36] while they indirectly mitigate measurement differences in this paper.

- Although piecewise monotonic data approximations indirectly mitigate the measurement differences in this paper, L1PMA, L2WPMA and L2CXCV can satisfactorily retrieve the theoretical SE properties of the indicative OV MV BPL topologies.

- The performance of piecewise monotonic data approximations strongly depends on the examined OV MV BPL topology, the maximum value $\alpha_{\text {CUD }}$ of the applied measurement difference CUD and the number of monotonic sections.

- As the mitigation performance of piecewise monotonic data approximations is examined with relation to the OV MV BPL topology, the presence of many short branches along the end-to-end transmission path entails a rich multipath environment. As already been mentioned in [33], [36], the spectral notches (extrema) that appear in the rich multipath environments (i.e., urban case) require additional monotonic sections so that the SE approximation may be accurate. Thanks to their adjustable number of monotonic sections, L1PMA and L2WMPA can be adaptive in order to improve their approximation efficiency by focusing on the improvement of SE $\triangle$ PES. Conversely, when OV MV BPL topologies of low number and long branches are examined (i.e., "LOS", rural and suburban case), L1PMA and L2WPMA need low number of monotonic sections so that high SE $\triangle \mathrm{PES}$ can be achieved. Also, L2CXCV, which does not take as input monotonic sections, can provide very good approximations of SE curves for specific OV MV BPL topologies.

To more elaborately examine the SE approximation by applying piecewise monotonic approximations, the relation among $\mathrm{SE}$ approximation accuracy, the maximum value $\alpha_{\text {CUD }}$, the applied piecewise monotonic data approximation and the number of monotonic 
sections should be investigated. As already been mentioned in Sec.3.1, SE $\triangle \mathrm{PES}$ acts as the main metric that assesses the mitigation efficiency of the piecewise monotonic data

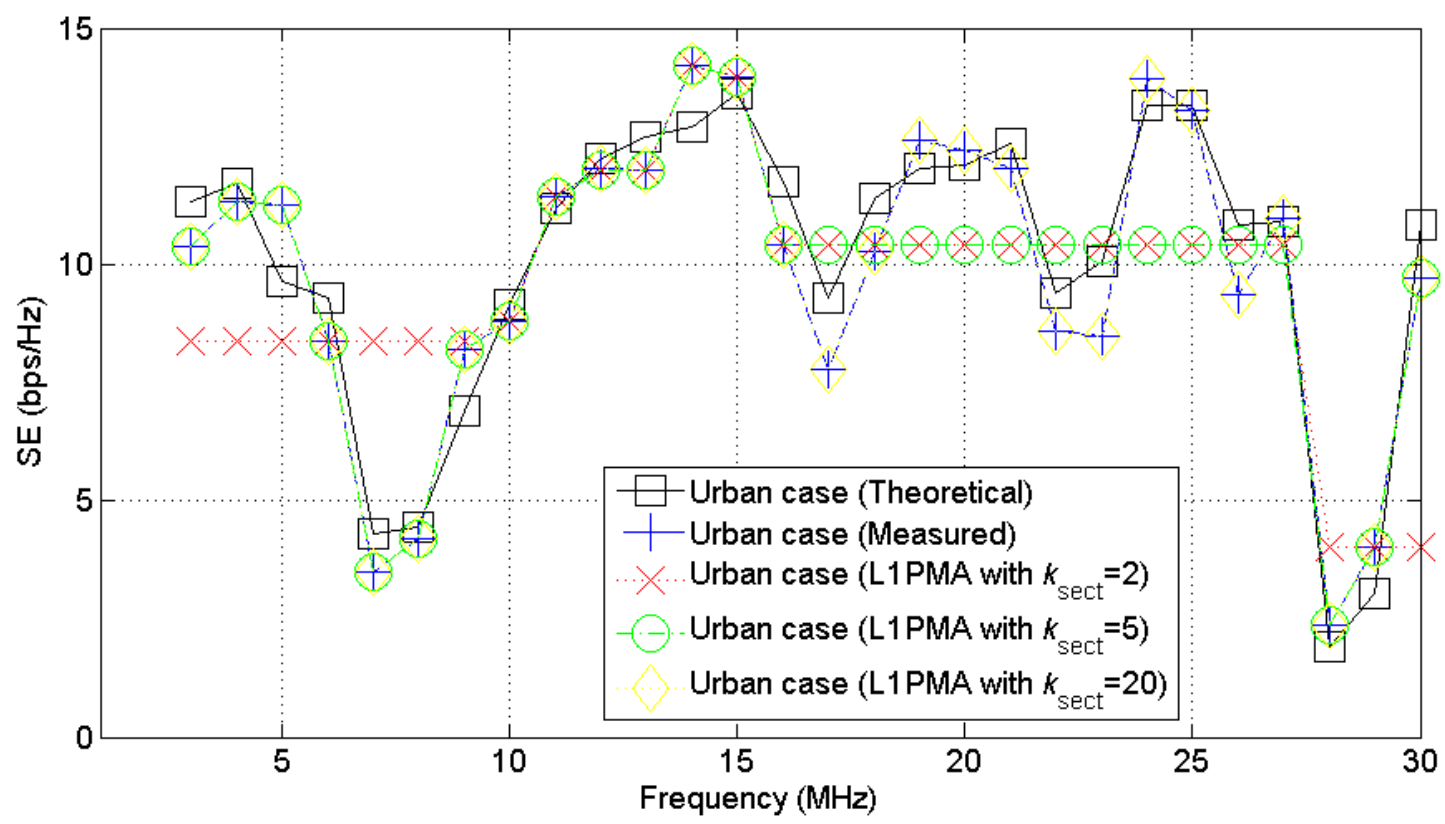

(a)

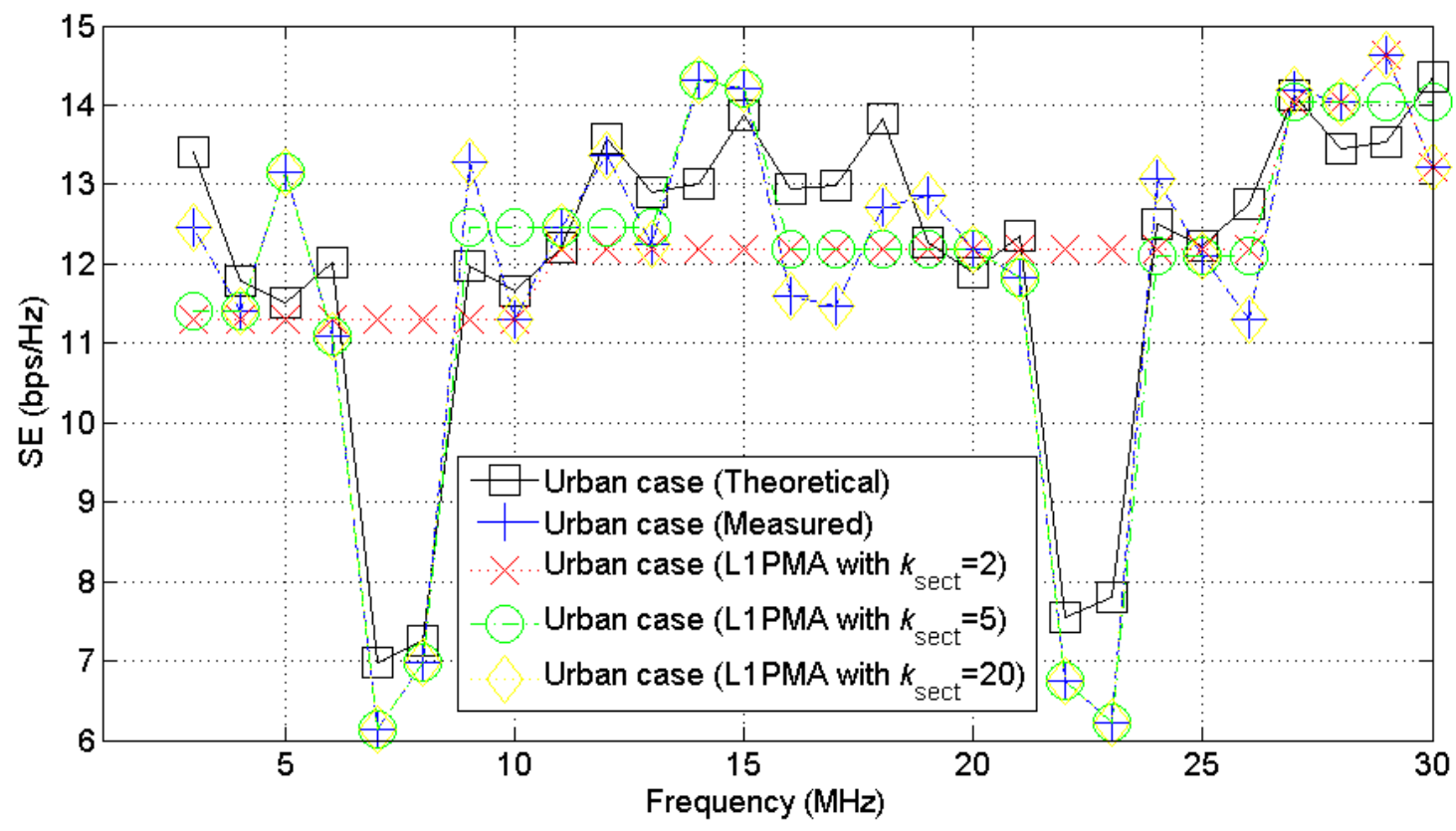

(b) 


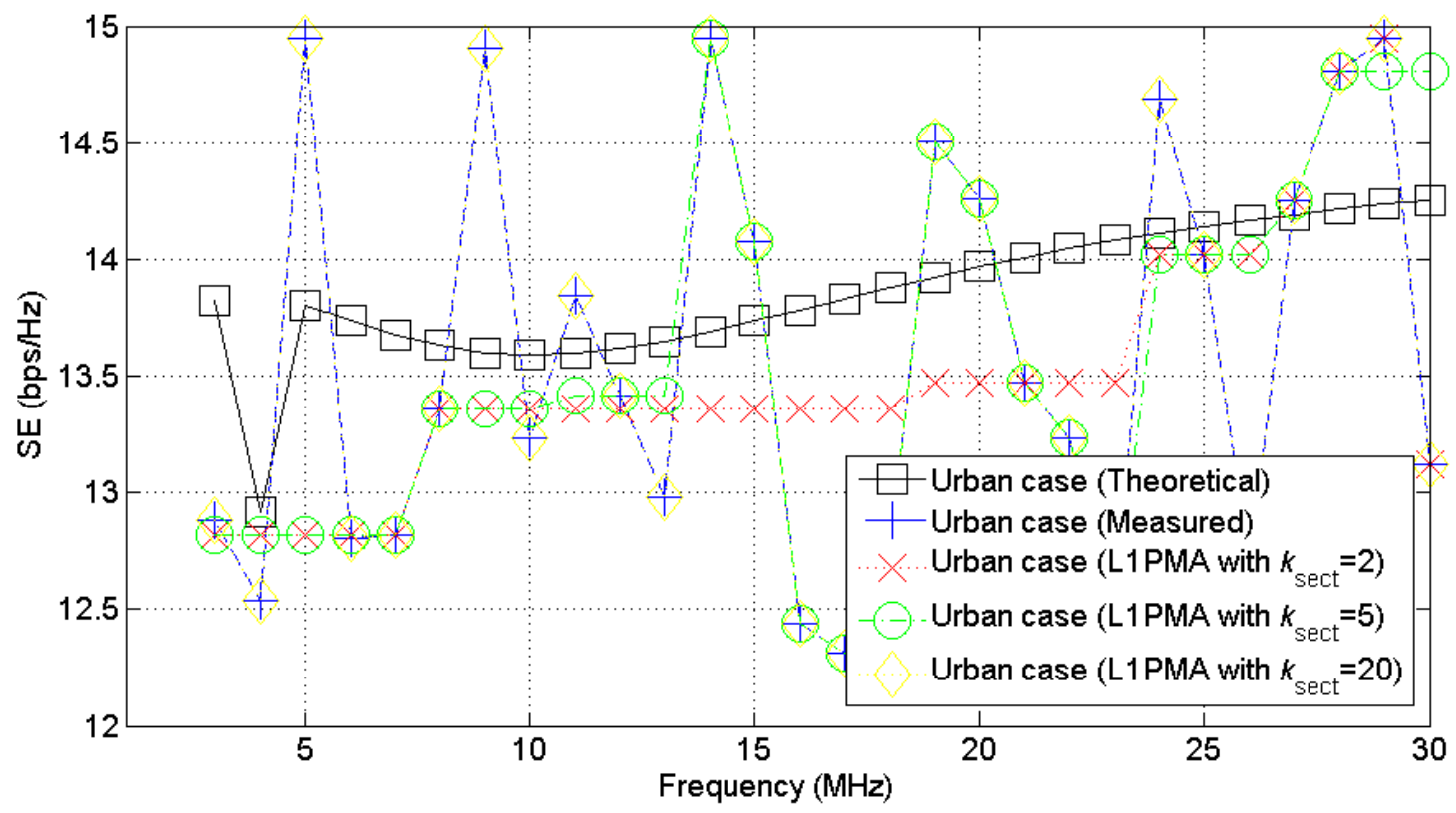

(c)

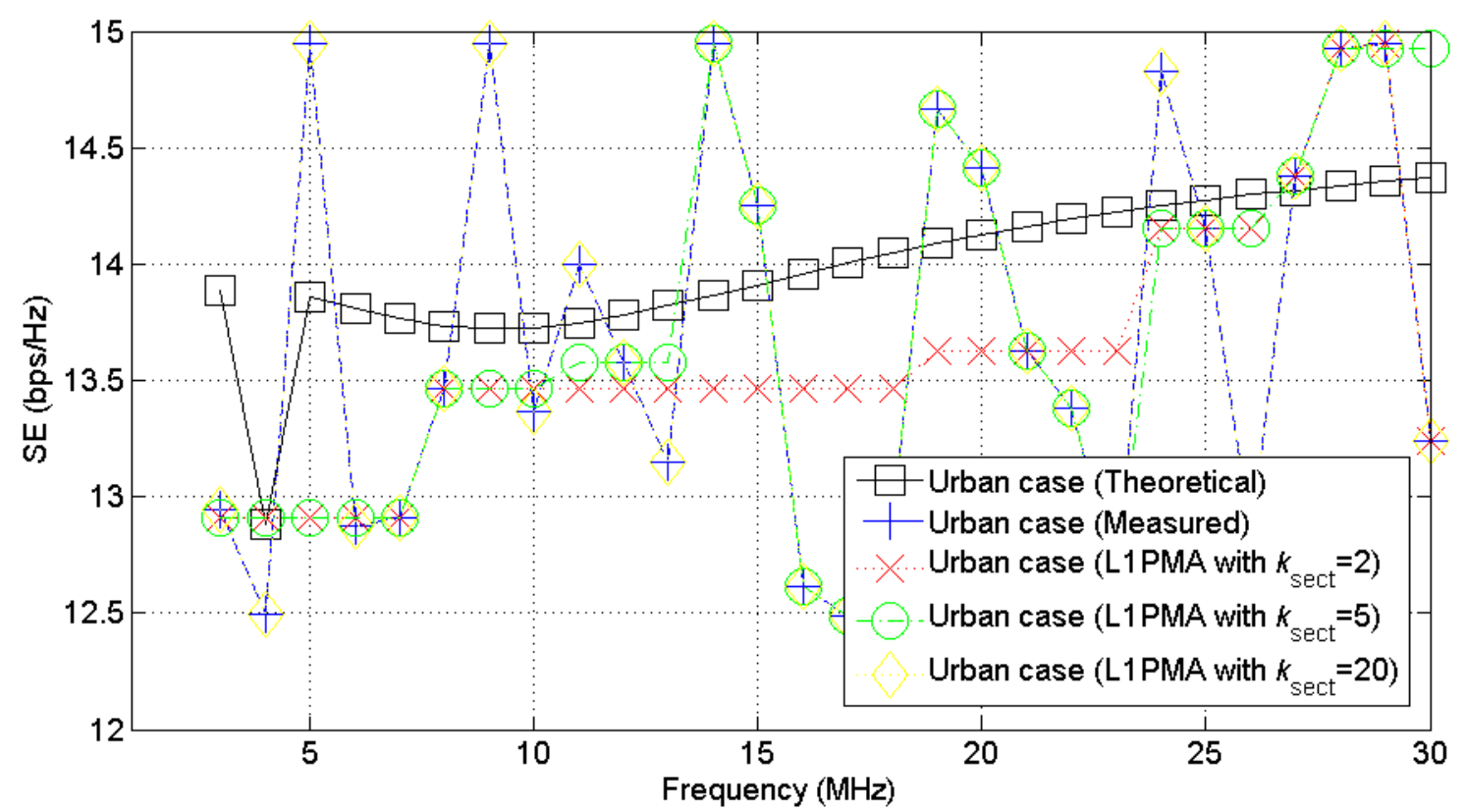

(d)

Fig. 1. Theoretical, measured and approximated SE of indicative OV MV BPL topologies when L1PMA is applied, measurement difference CUD of $\alpha_{\mathrm{CUD}}=5 \mathrm{~dB}$ is assumed. Three representative cases of monotonic sections (i.e, $k=1,2$ and 20) are assumed for the SE approximation. (a) Urban case. (b) Suburban case. (c) Rural case. (d) "LOS" case. 


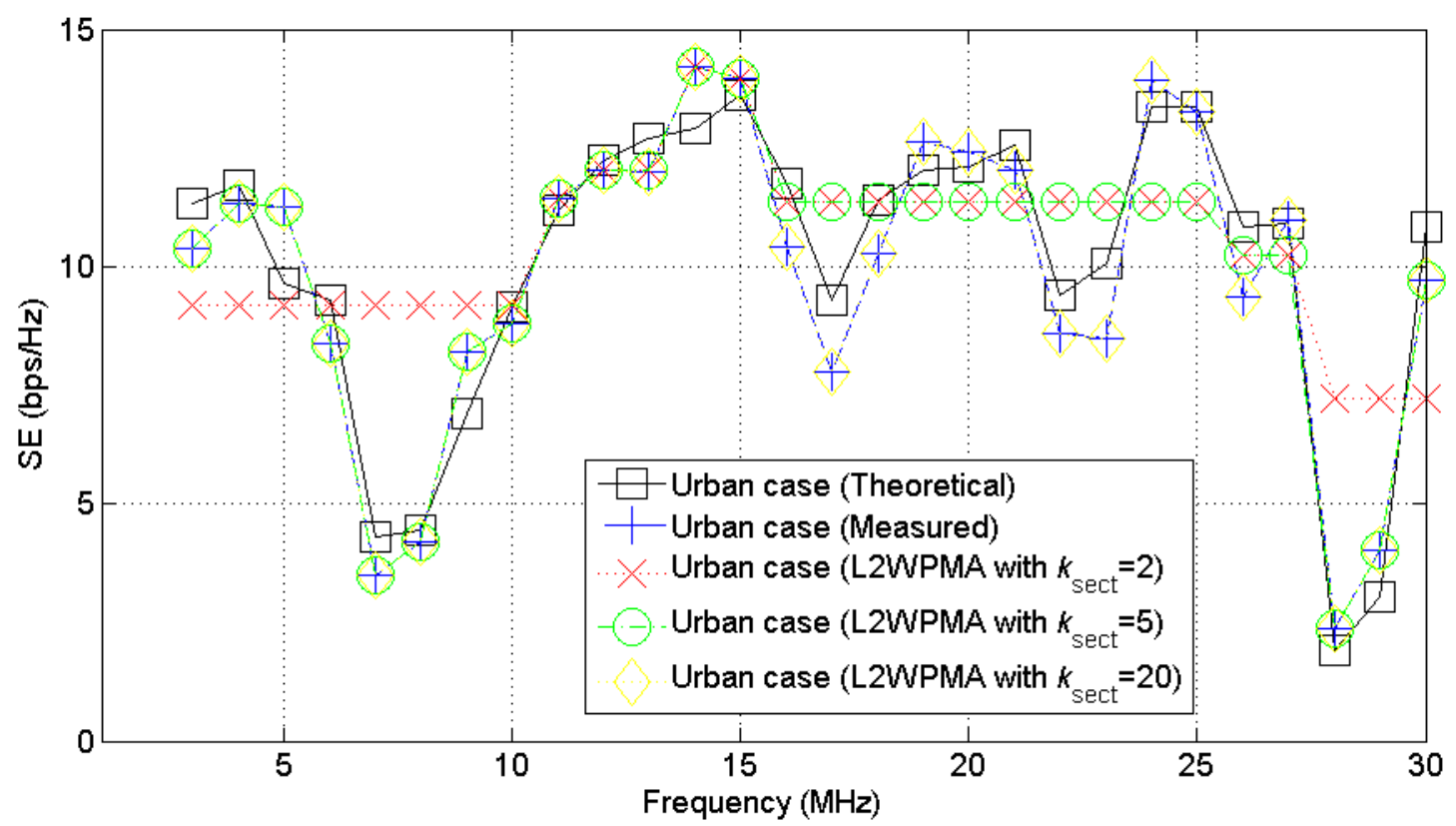

(a)

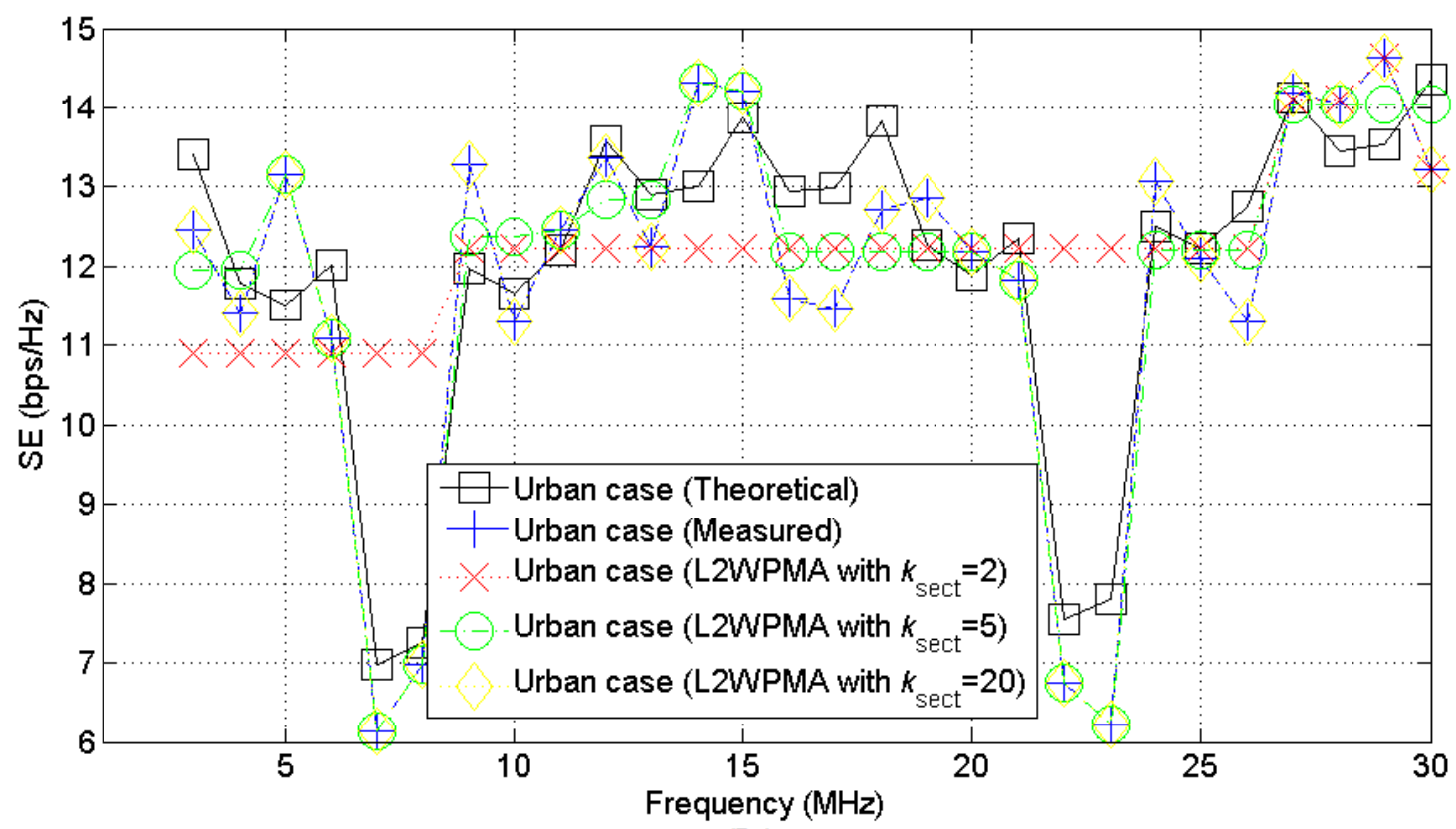

(b) 


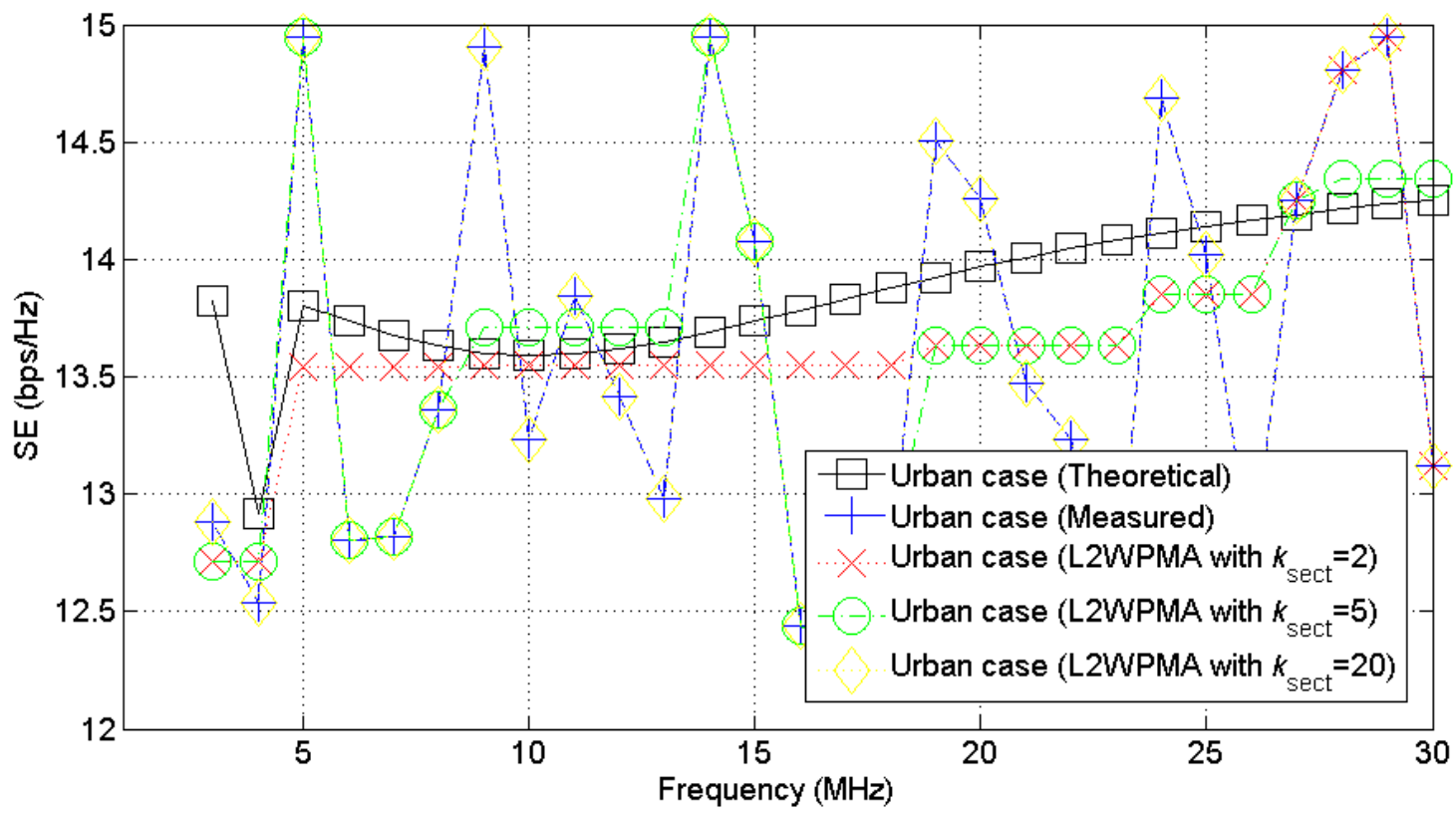

(c)

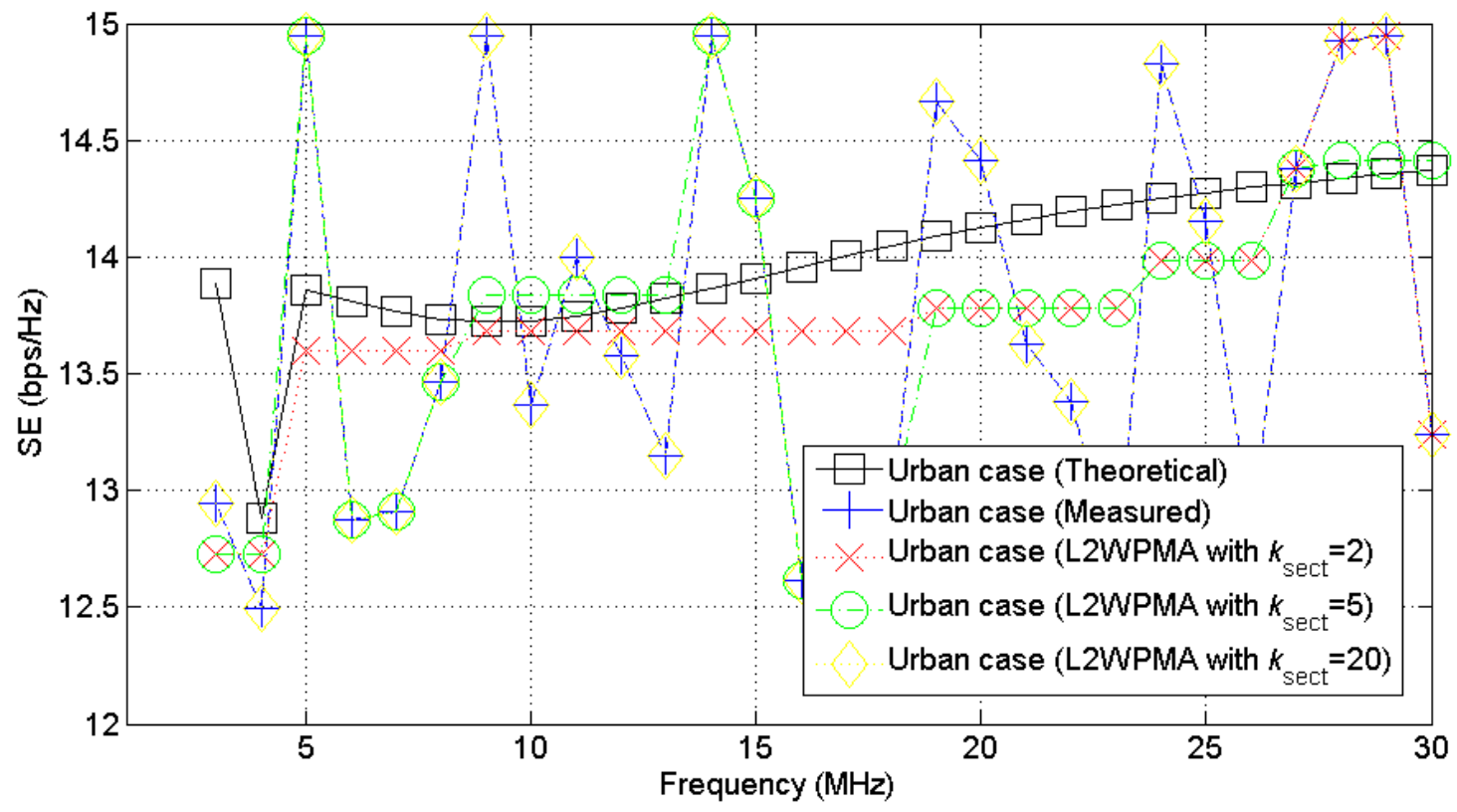

(d)

Fig. 2. Same curves with Fig.1 but for L2WPMA. 


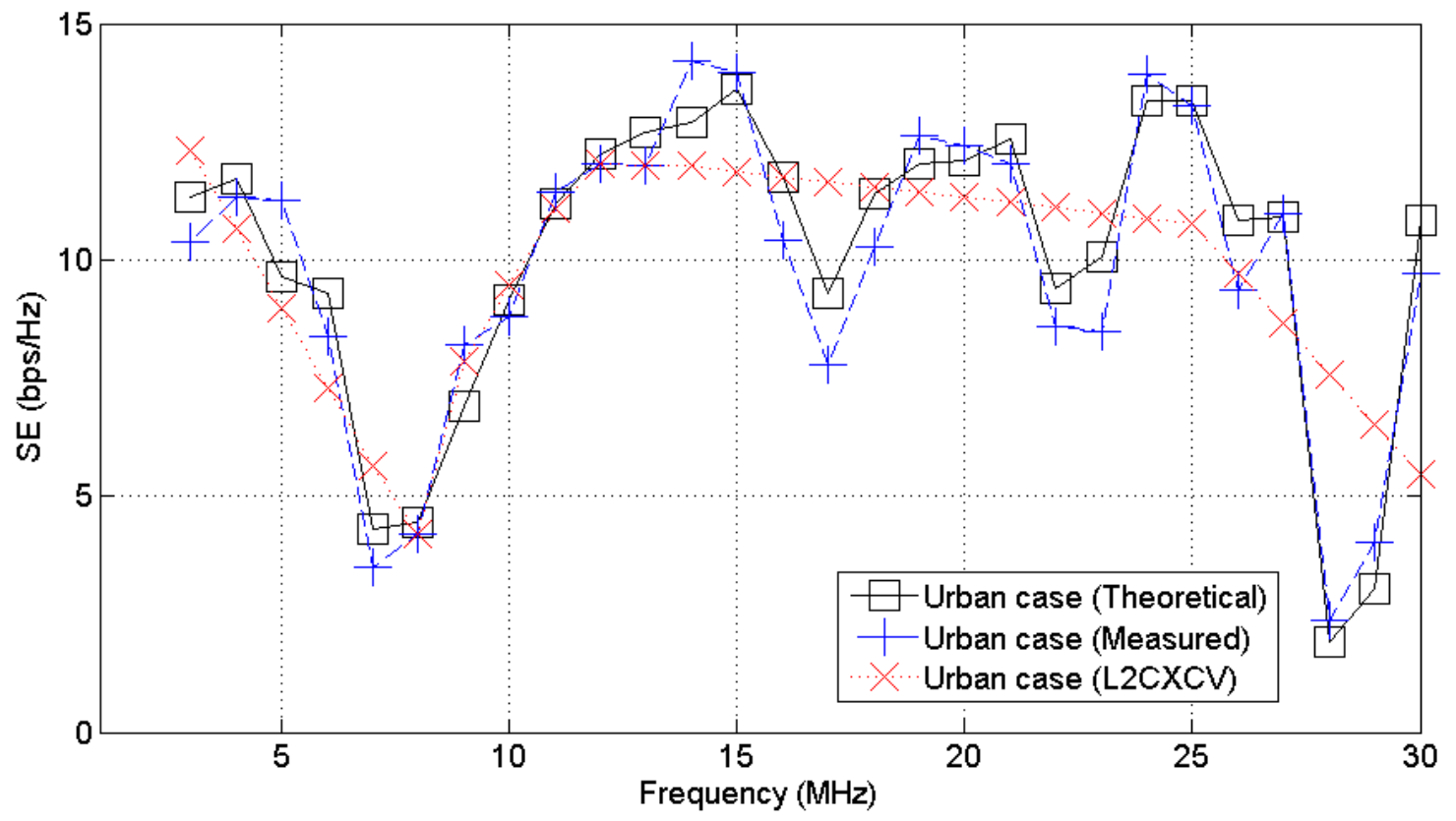

(a)

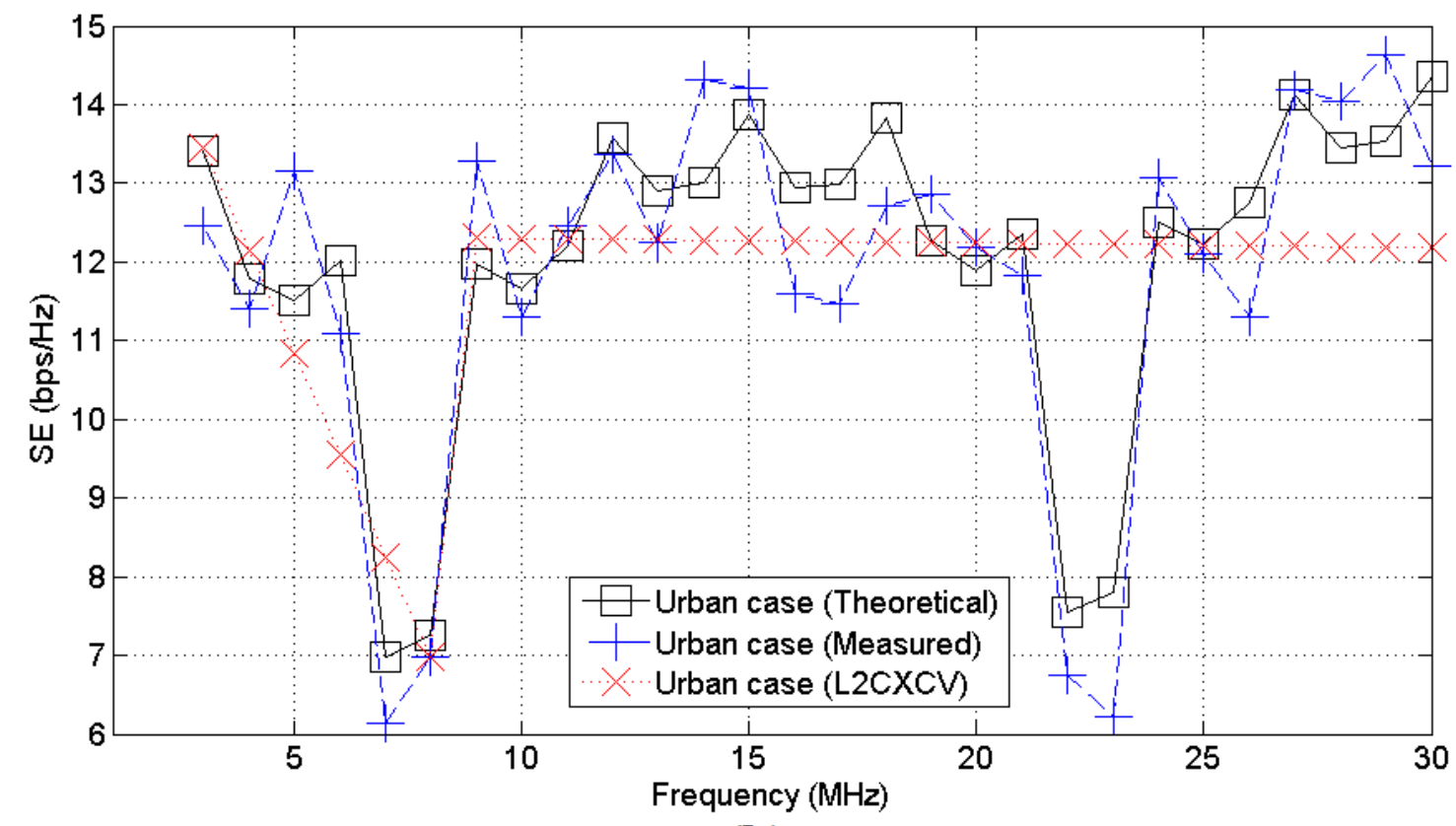

(b) 

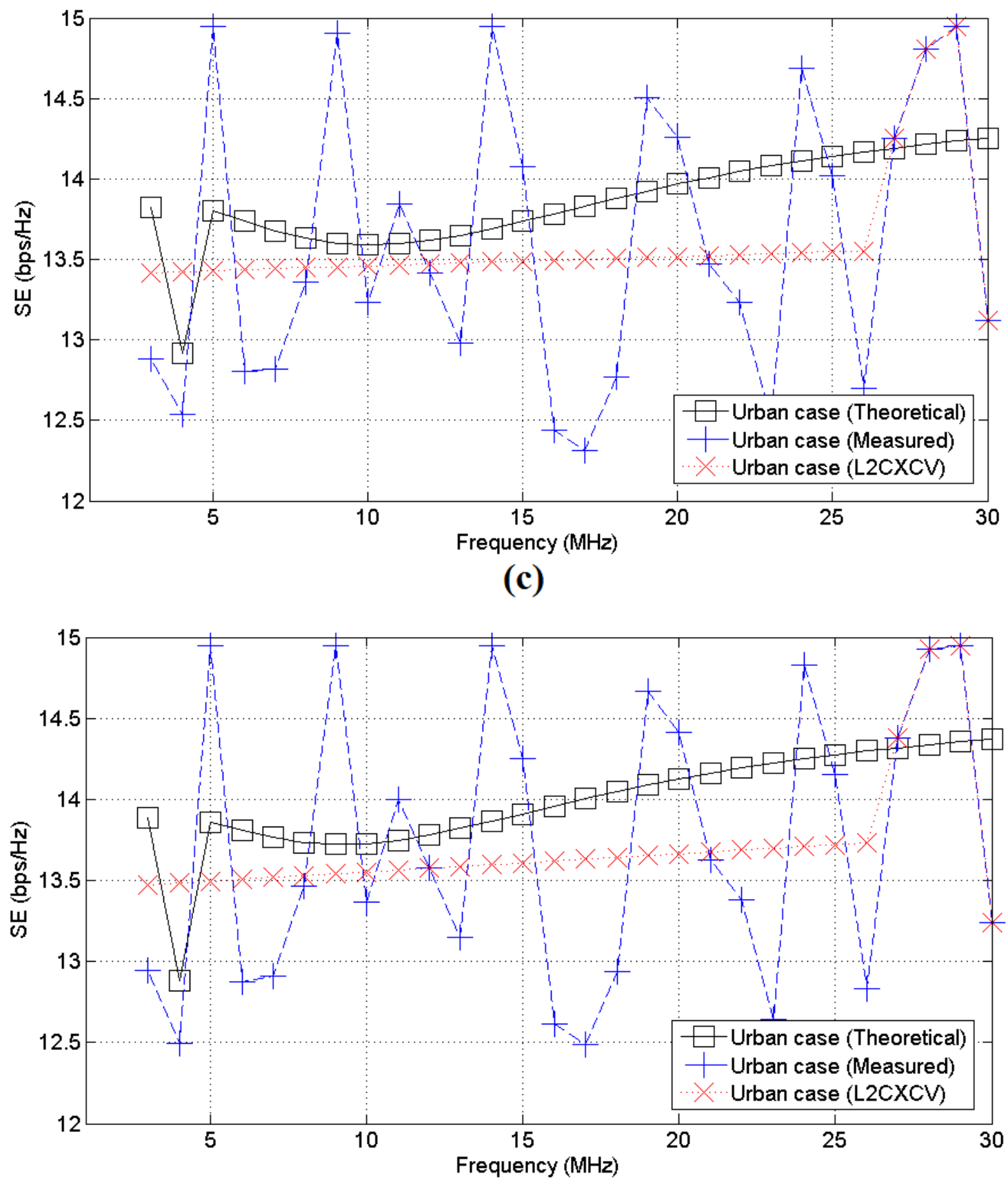

(d)

Fig. 3. Same curves with Fig. 1 but for L2CXCV.

approximations. For the Figs. 1-3, the SE $\triangle \mathrm{PES}$ curves of the applied piecewise monotonic data approximations are given in Figs. 4-6, respectively, when various measurement difference CUDs and number of monotonic sections are considered. In fact, maximum value $\alpha_{\mathrm{CUD}}$ that ranges from 0 to $5 \mathrm{~dB}$ is assumed. On the basis of Figs. 4-6, the maximum SE $\triangle \mathrm{PES}$ and the corresponding number of monotonic sections are reported in Tables 1-3 when L1PMA, L2WPMA and L2CXCV are applied, respectively. 


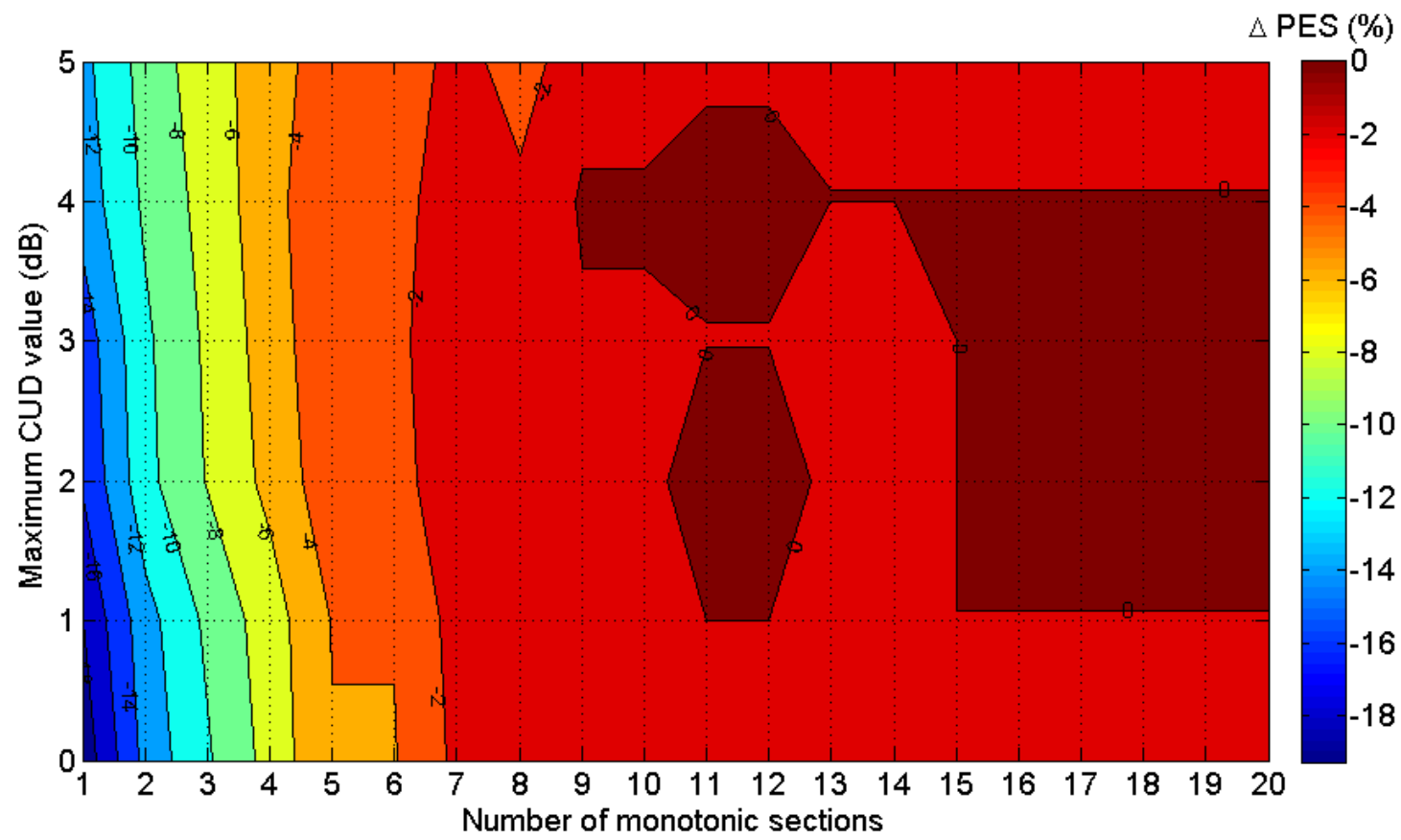

(a)

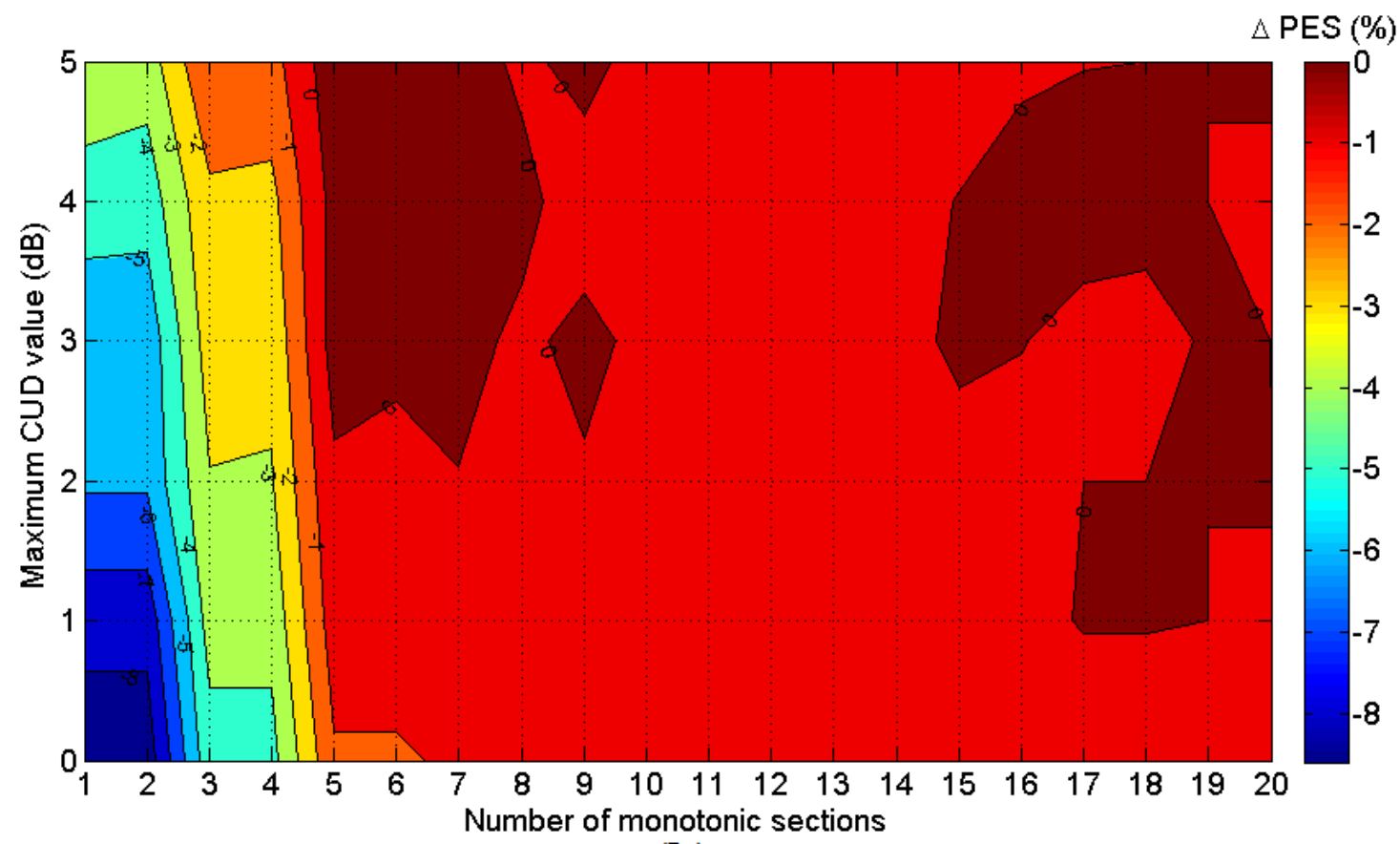

(b) 


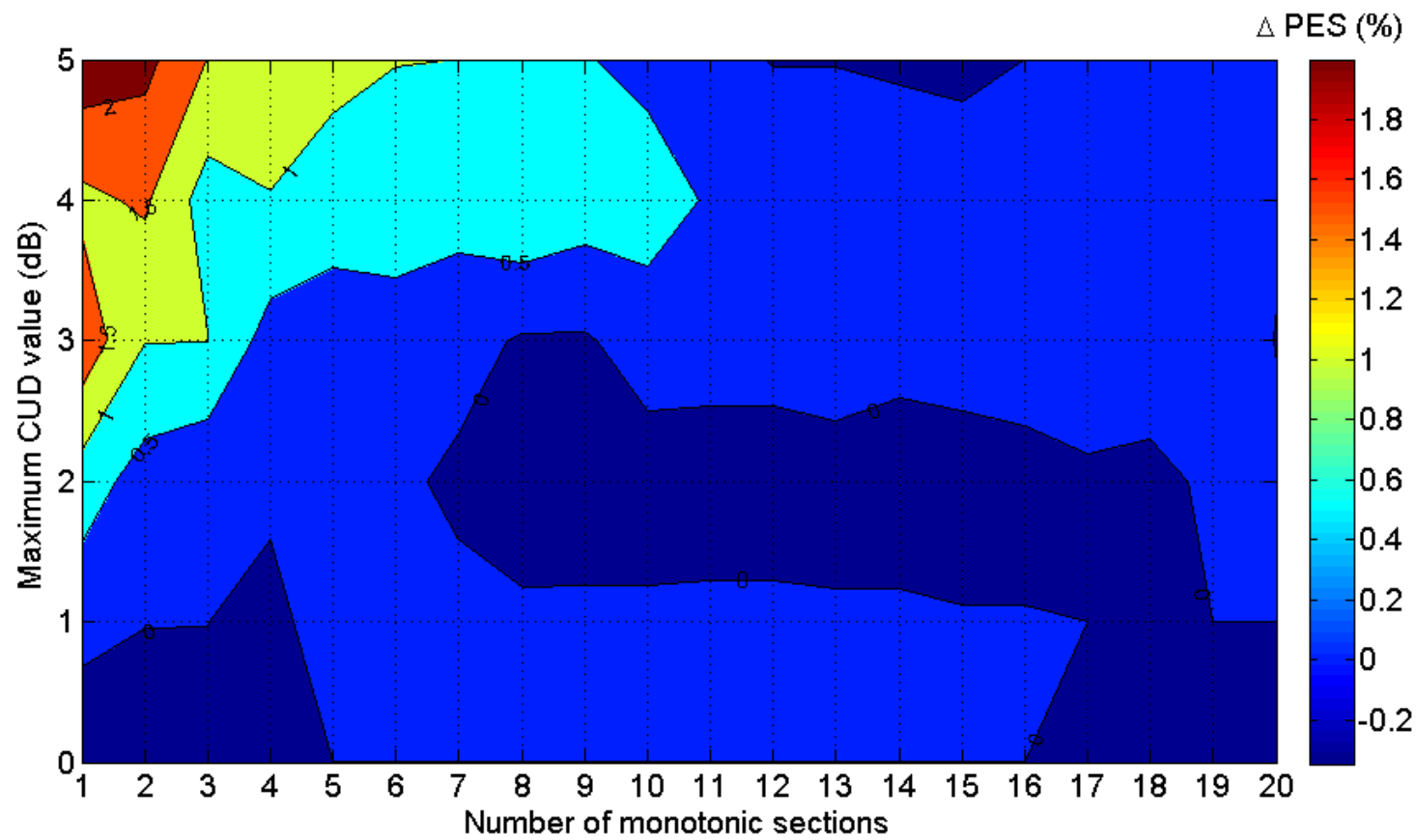

(c)

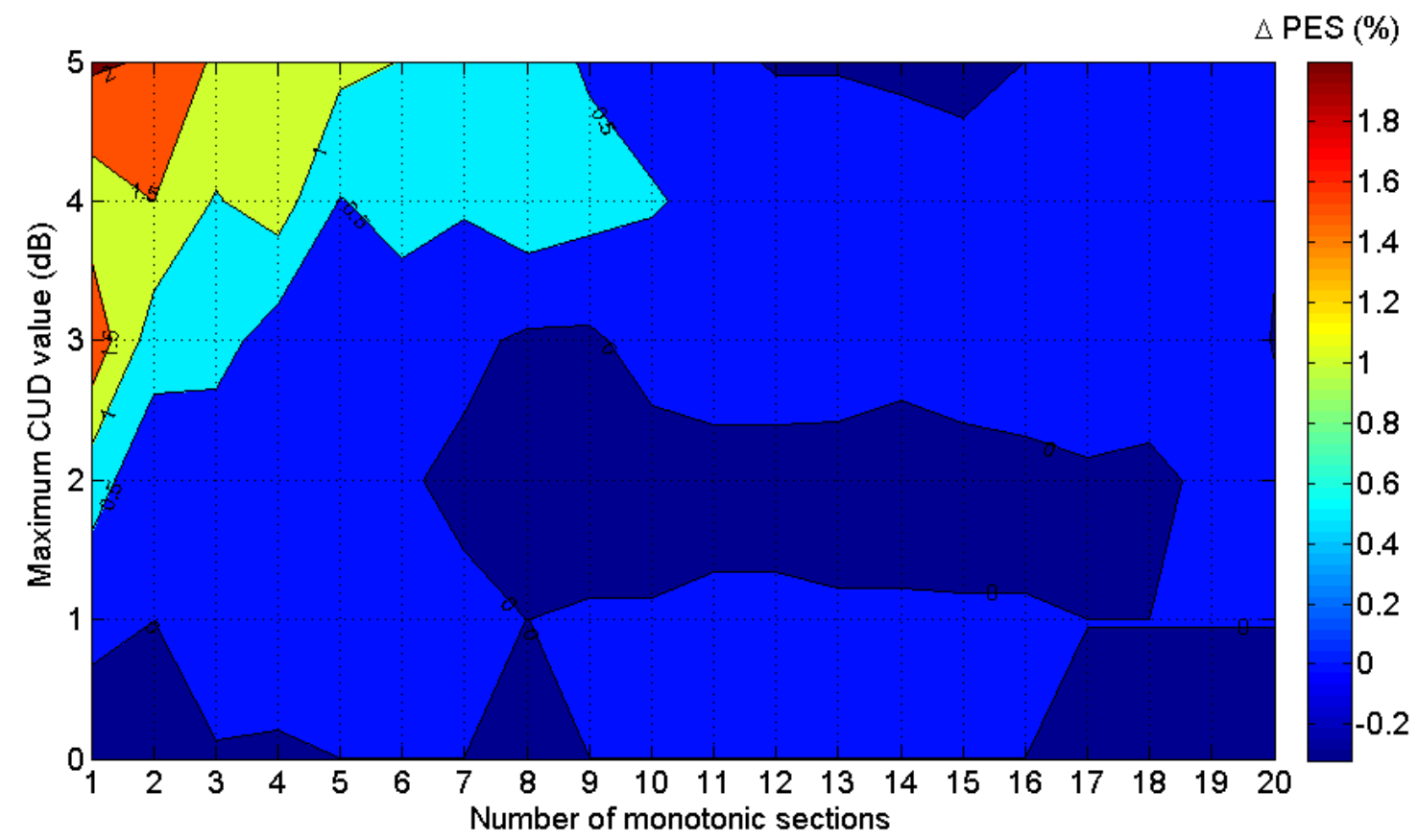

(d)

Fig. 4. SE $\triangle \mathrm{PES}$ of indicative $\mathrm{OV}$ MV BPL topologies when L1PMA is applied and measurement difference CUD of $\alpha \mathrm{CUD}=5 \mathrm{~dB}$ is assumed. Three representative cases of monotonic sections (i.e, $\mathrm{k}=1,2$ and 20) are assumed for the SE approximation -see also Figs. 1(a)-(d)-. (a) Urban case. (b) Suburban case. (c) Rural case. (d) "LOS" case. 


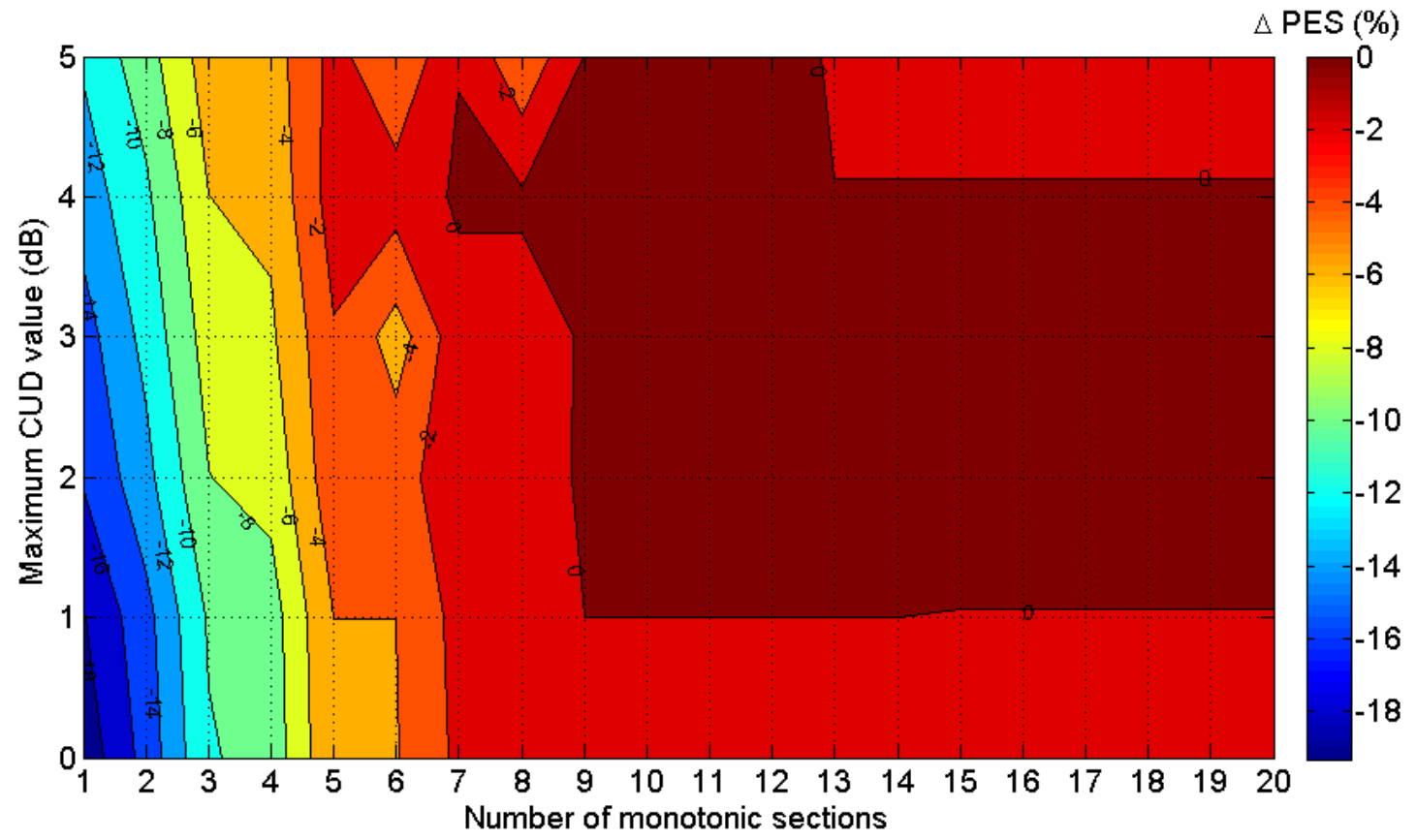

(a)

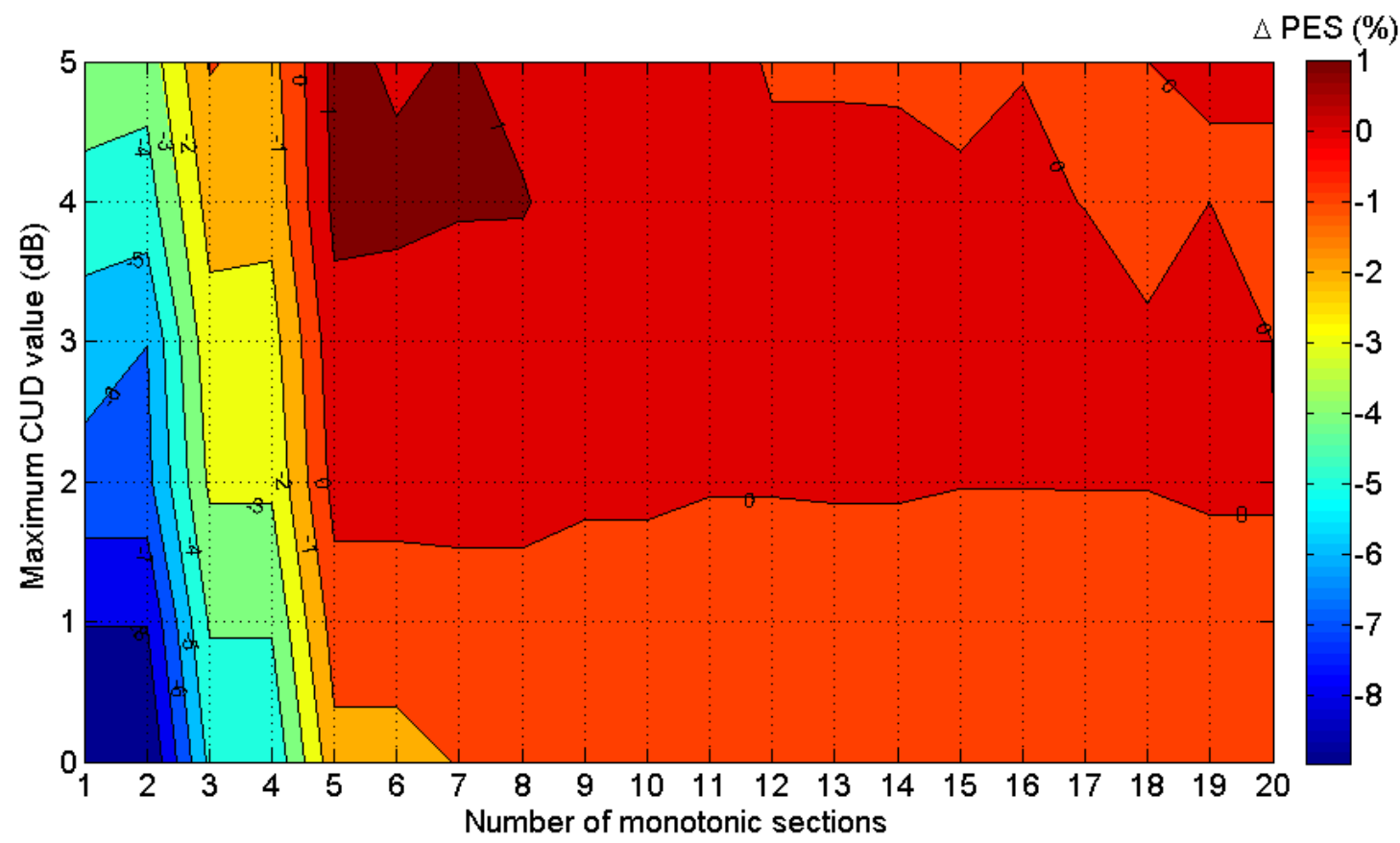

(b) 


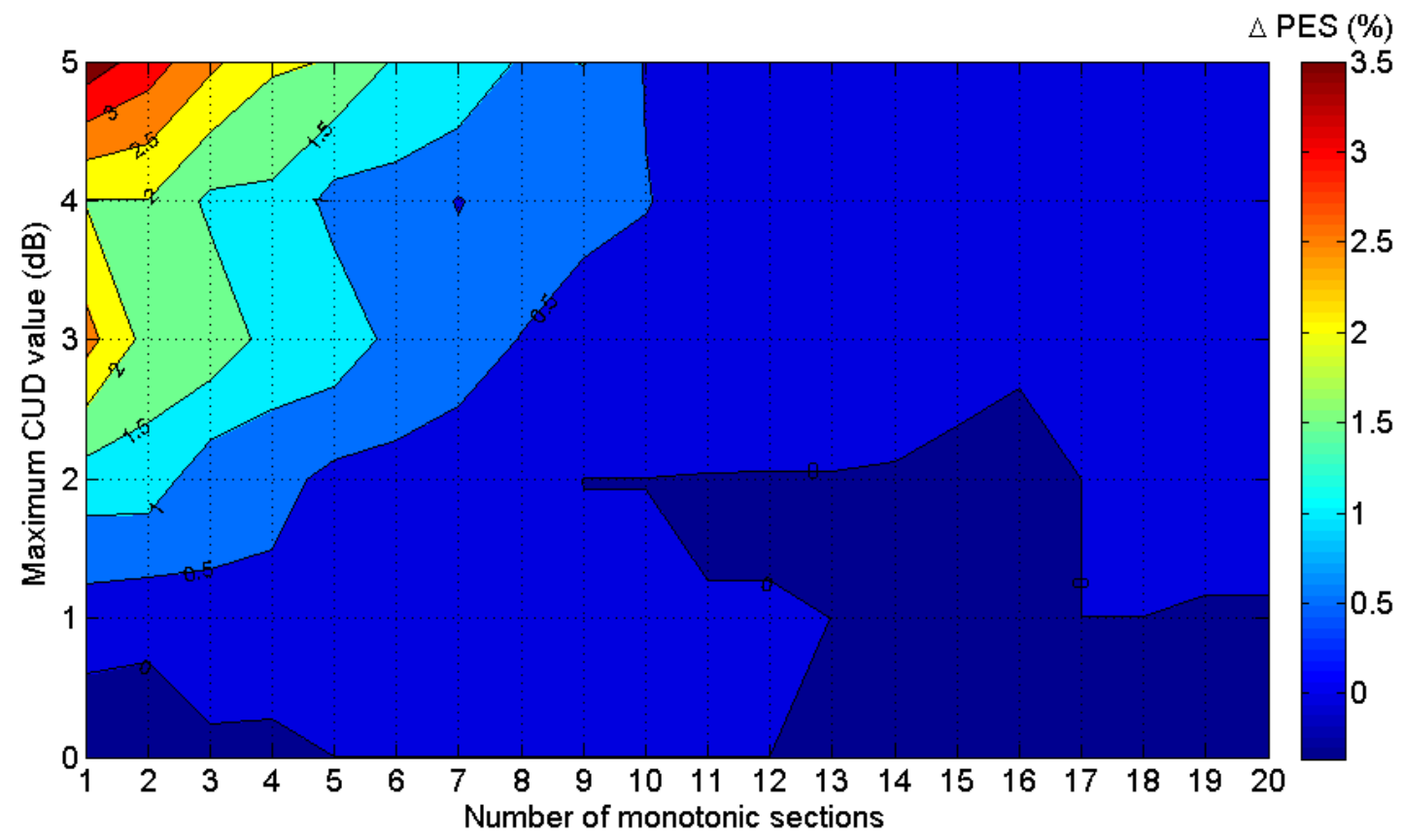

(c)

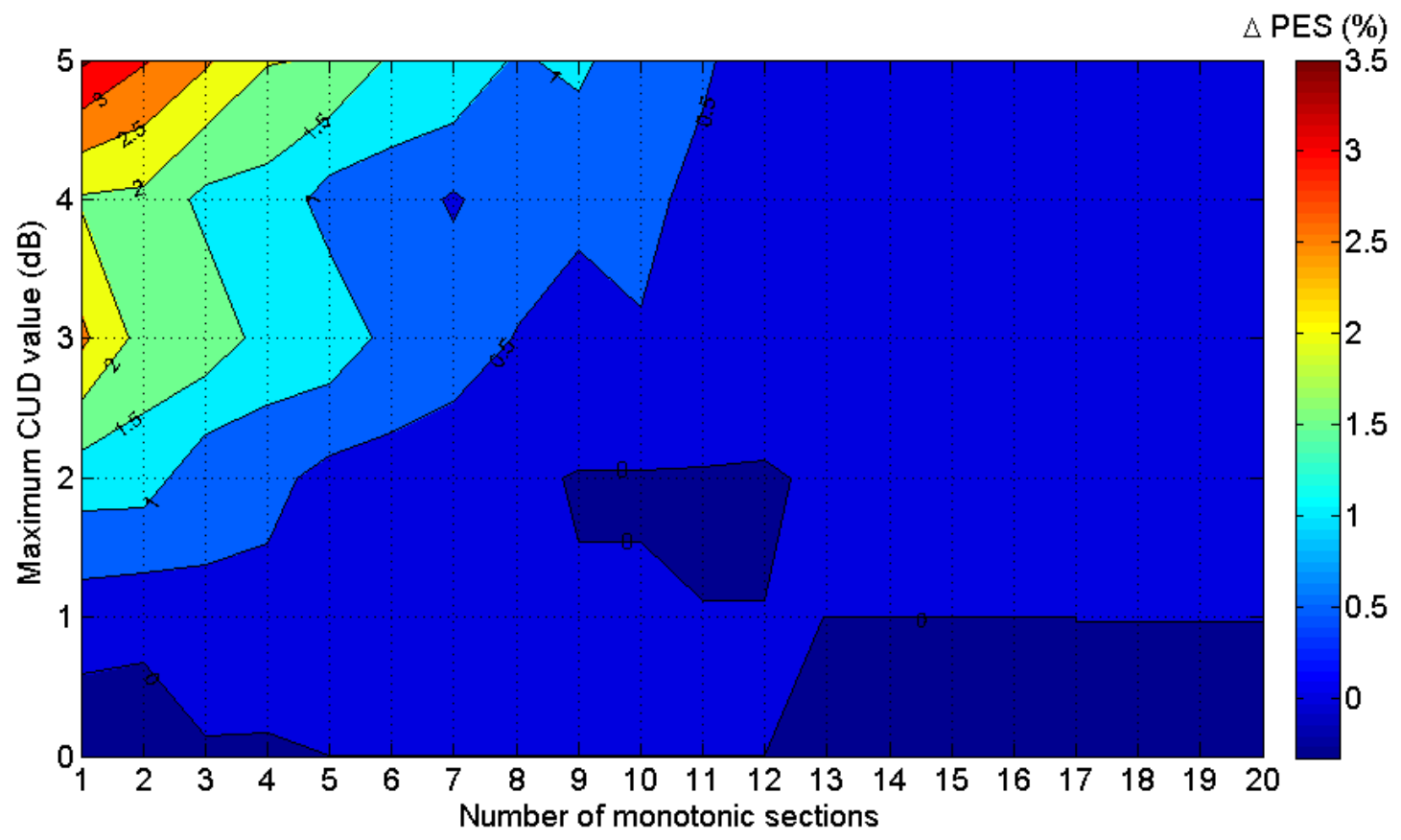

(d)

Fig. 5. Same curves with Fig.4 but for L2WPMA -see also Figs. 2(a)-(d)-. 


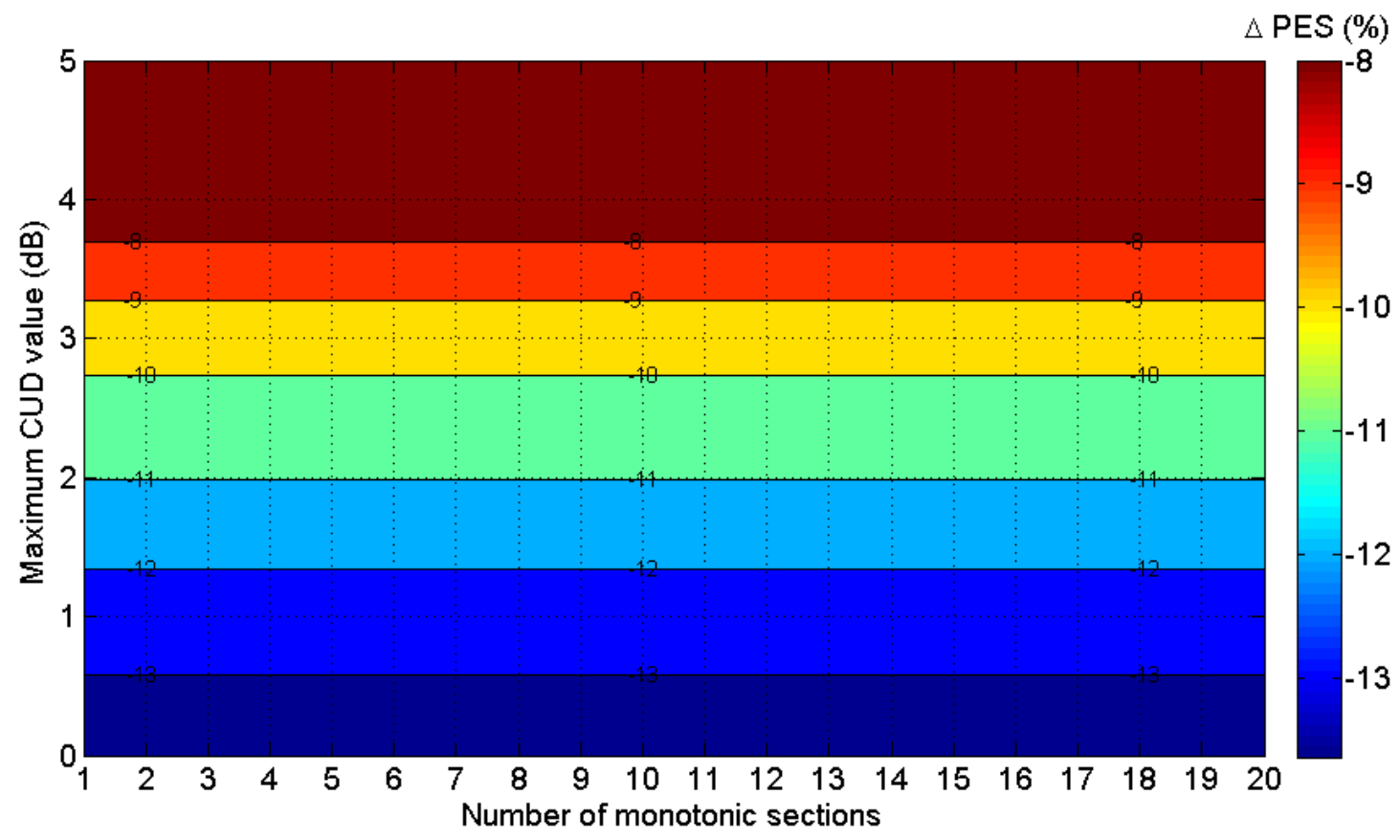

(a)

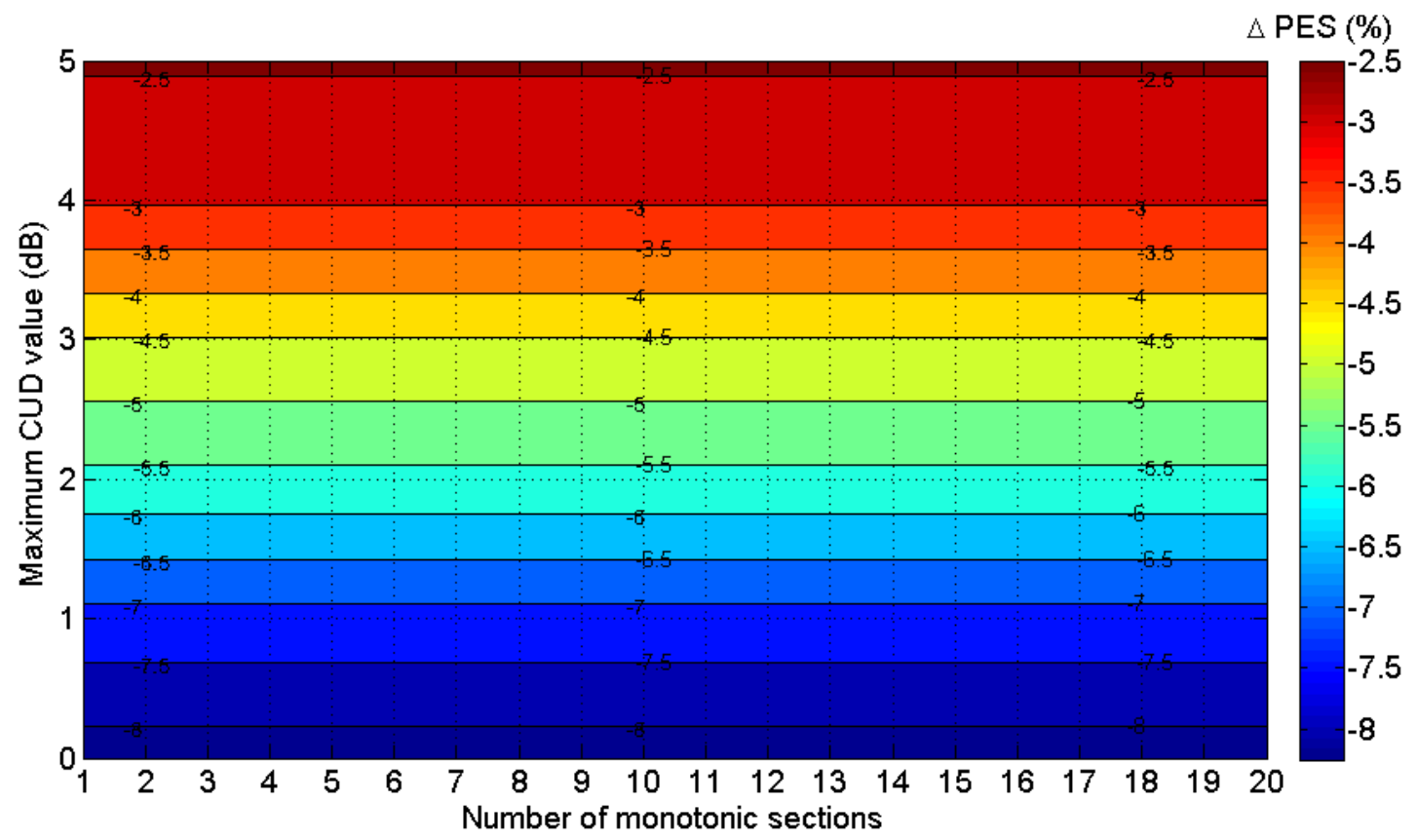

(b) 


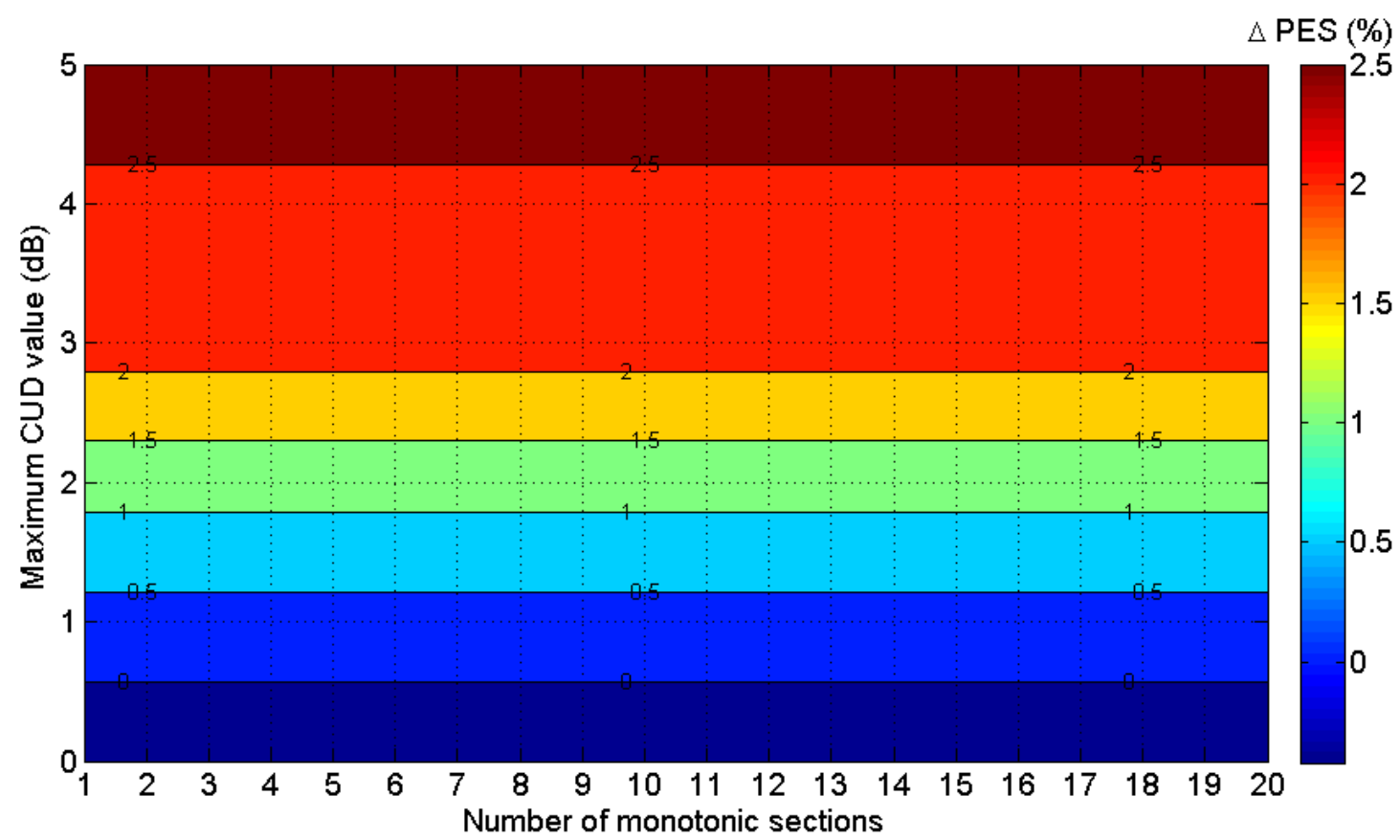

(c)

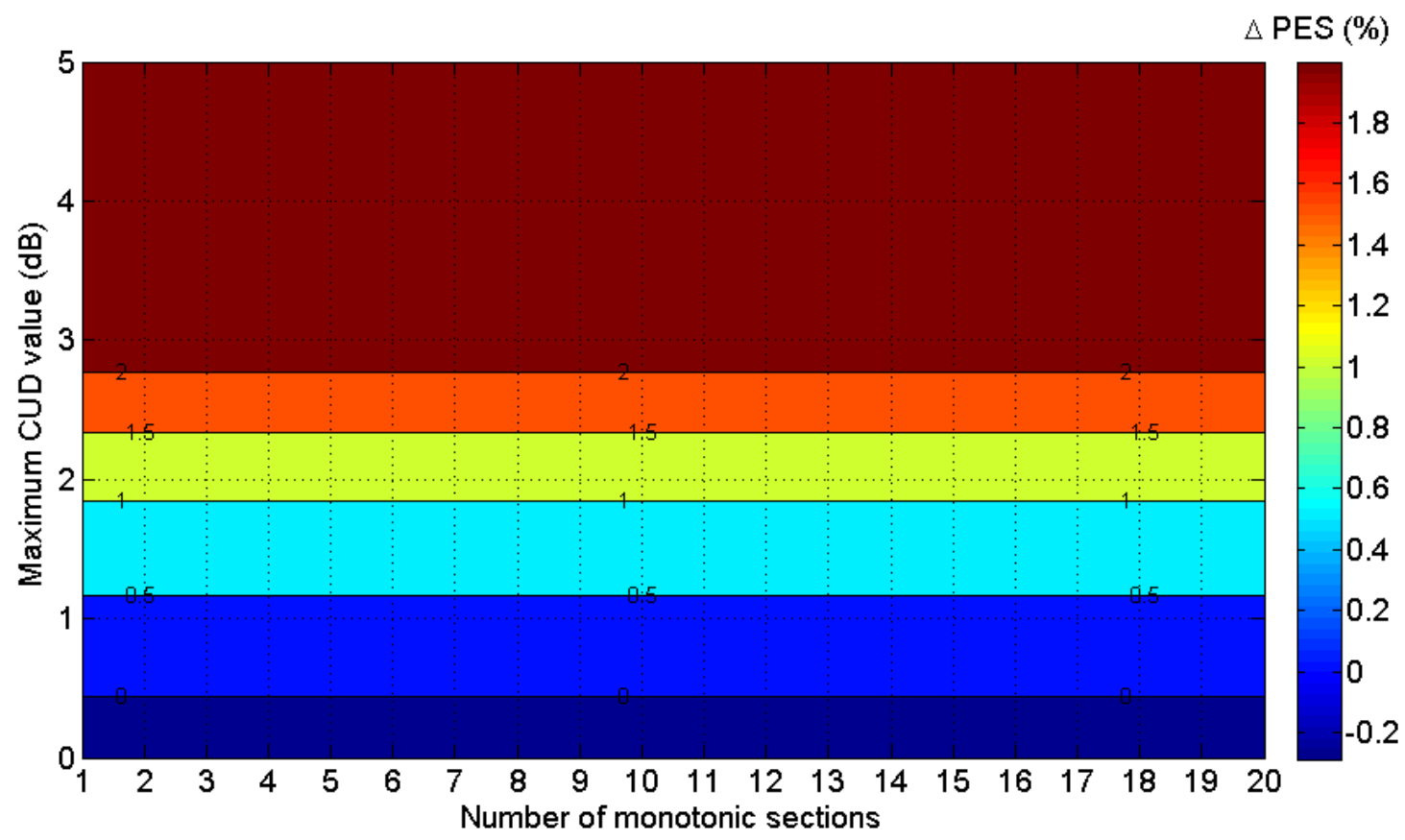

(d)

Fig. 6. Same curves with Fig.4 but for L2CXCV -see also Figs. 3(a)-(d)-. 
Table 1. Maximum $\triangle \mathrm{PES}$ and Corresponding Number of Monotonic Sections when L1PMA is Applied

\begin{tabular}{|c|c|c|c|c|c|c|c|c|}
\hline \multirow{3}{*}{$\begin{array}{l}\text { Maximum } \\
\text { Value } \alpha_{C U D} \\
\text { (dB) }\end{array}$} & \multicolumn{8}{|c|}{ Indicative OV MV BPL Topologies } \\
\hline & \multicolumn{2}{|c|}{ Urban Case A } & \multicolumn{2}{|c|}{ Suburban Case } & \multicolumn{2}{|c|}{ Rural Case } & \multicolumn{2}{|c|}{ "LOS" Case } \\
\hline & $\begin{array}{c}\text { SE } \triangle \mathrm{PES} \\
(\%)\end{array}$ & $\begin{array}{c}\text { Number of } \\
\text { Monotonic } \\
\text { Sections }\end{array}$ & $\begin{array}{c}\text { SE } \triangle \mathrm{PES} \\
(\%)\end{array}$ & $\begin{array}{c}\text { Number of } \\
\text { Monotonic } \\
\text { Sections }\end{array}$ & $\begin{array}{c}\text { SE } \triangle \mathrm{PES} \\
(\%)\end{array}$ & $\begin{array}{l}\text { Number of } \\
\text { Monotonic } \\
\text { Sections }\end{array}$ & $\begin{array}{c}\text { SE } \triangle \mathrm{PES} \\
(\%)\end{array}$ & $\begin{array}{c}\text { Number of } \\
\text { Monotonic } \\
\text { Sections }\end{array}$ \\
\hline 0 & $-1.92 \times 10^{-5}$ & 11 & $-2.09 \times 10^{-5}$ & 19 & $-1.71 \times 10^{-5}$ & 5 & $-1.72 \times 10^{-5}$ & 5 \\
\hline 1 & $-5.27 \times 10^{-7}$ & 9 & $7.7 \times 10^{-3}$ & 17 & 0.23 & 5 & 0.41 & 3 \\
\hline 2 & 0.09 & 11 & $1.47 \times 10^{-6}$ & 17 & 0.76 & 1 & 0.71 & 1 \\
\hline 3 & $3.18 \times 10^{-6}$ & 15 & 0.45 & 5 & 1.85 & 1 & 1.89 & 1 \\
\hline 4 & 0.17 & 9 & 0.34 & 5 & 1.58 & 2 & 1.50 & 2 \\
\hline 5 & $-4.97 \times 10^{-6}$ & 13 & 0.68 & 5 & 2.33 & 1 & 2.09 & 1 \\
\hline
\end{tabular}

Table 2. Maximum $\triangle \mathrm{PES}$ and Corresponding Number of Monotonic Sections when L2WPMA is Applied

\begin{tabular}{|c|c|c|c|c|c|c|c|c|}
\hline \multirow{3}{*}{$\begin{array}{c}\text { Maximum } \\
\text { Value } \\
\alpha_{\text {CUD }} \\
\text { (dB) }\end{array}$} & \multicolumn{8}{|c|}{ Indicative OV MV BPL Topologies } \\
\hline & \multicolumn{2}{|c|}{ Urban Case A } & \multicolumn{2}{|c|}{ Suburban Case } & \multicolumn{2}{|c|}{ Rural Case } & \multicolumn{2}{|c|}{ "LOS" Case } \\
\hline & $\begin{array}{c}\text { SE } \triangle \mathrm{PES} \\
(\%)\end{array}$ & $\begin{array}{c}\text { Number of } \\
\text { Monotonic } \\
\text { Sections }\end{array}$ & $\begin{array}{c}\text { SE } \triangle \mathrm{PES} \\
(\%)\end{array}$ & $\begin{array}{c}\text { Number of } \\
\text { Monotonic } \\
\text { Sections }\end{array}$ & $\begin{array}{c}\text { SE } \triangle \mathrm{PES} \\
(\%)\end{array}$ & $\begin{array}{c}\text { Number of } \\
\text { Monotonic } \\
\text { Sections }\end{array}$ & $\begin{array}{c}\text { SE } \triangle \mathrm{PES} \\
(\%)\end{array}$ & $\begin{array}{c}\text { Number of } \\
\text { Monotonic } \\
\text { Sections } \\
\end{array}$ \\
\hline 0 & $-1.86 \times 10^{-5}$ & 11 & $-2.15 \times 10^{-5}$ & 19 & $-1.72 \times 10^{-5}$ & 5 & $-1.71 \times 10^{-5}$ & 5 \\
\hline 1 & $-4.30 \times 10^{-7}$ & 9 & $-3.07 \times 10^{-6}$ & 19 & 0.41 & 3 & 0.42 & 3 \\
\hline 2 & 0.23 & 9 & 0.41 & 7 & 1.28 & 1 & 1.24 & 1 \\
\hline 3 & 0.14 & 9 & 0.67 & 5 & 2.69 & 1 & 2.62 & 1 \\
\hline 4 & 0.71 & 9 & 1.23 & 5 & 1.98 & 2 & 1.95 & 1 \\
\hline 5 & 0.09 & 9 & 1.27 & 5 & 3.81 & 1 & 3.59 & 1 \\
\hline
\end{tabular}

Table 3. Maximum $\triangle \mathrm{PES}$ and Corresponding Number of Monotonic Sections when L2CXCV is Applied

\begin{tabular}{|c|c|c|c|c|c|c|c|c|}
\hline \multirow{3}{*}{$\begin{array}{l}\text { Maximum } \\
\text { Value } \\
\text { oCUD } \\
\text { (dB) }\end{array}$} & \multicolumn{8}{|c|}{ Indicative OV MV BPL Topologies } \\
\hline & \multicolumn{2}{|c|}{ Urban Case A } & \multicolumn{2}{|c|}{ Suburban Case } & \multicolumn{2}{|c|}{ Rural Case } & \multicolumn{2}{|c|}{ "LOS" Case } \\
\hline & $\begin{array}{c}\mathrm{SE} \\
\Delta \mathrm{PES} \\
(\%)\end{array}$ & $\begin{array}{c}\text { Number of } \\
\text { Monotonic } \\
\text { Sections }\end{array}$ & $\begin{array}{c}\mathrm{SE} \\
\Delta \mathrm{PES} \\
(\%)\end{array}$ & $\begin{array}{l}\text { Number of } \\
\text { Monotonic } \\
\text { Sections }\end{array}$ & $\begin{array}{c}\mathrm{SE} \\
\Delta \mathrm{PES} \\
(\%)\end{array}$ & $\begin{array}{l}\text { Number of } \\
\text { Monotonic } \\
\text { Sections }\end{array}$ & $\begin{array}{c}\mathrm{SE} \\
\Delta \mathrm{PES} \\
(\%)\end{array}$ & $\begin{array}{c}\text { Number of } \\
\text { Monotonic } \\
\text { Sections }\end{array}$ \\
\hline 0 & -13.64 & - & -8.25 & - & -0.42 & - & -0.29 & - \\
\hline 1 & -15.53 & - & -7.16 & - & 0.3185 & - & 0.38 & - \\
\hline 2 & -10.98 & - & -5.61 & - & 1.18 & - & 1.11 & - \\
\hline 3 & -9.65 & - & -4.52 & - & 2.22 & - & 2.27 & - \\
\hline 4 & -7.26 & - & -2.93 & - & 2.42 & - & 2.19 & - \\
\hline 5 & -7.15 & - & -2.44 & - & 2.70 & - & 2.47 & - \\
\hline
\end{tabular}

From Figs. 4-6 and Tables 1-3, certain remarks can be reported that characterize the application behavior of piecewise monotonic data approximations for the various OV MV BPL topologies. More specifically:

- When urban OV MV BPL topologies are examined, piecewise monotonic data approximations cannot satisfactorily mitigate the added measurement differences. Actually, when the assumed maximum value $\alpha_{\mathrm{CUD}}$ remains low (i.e., below $2 \mathrm{~dB}$ ), all the applied piecewise monotonic data approximations of this paper cannot provide a clearly better SE approximation than the measured SE since SE $\triangle \mathrm{PES}$ remains marginally lower than zero in these cases. In contrast, when the assumed maximum value $\alpha_{\text {CUD }}$ becomes high (i.e., above 2dB), L2WPMA provides SE approximations that are better than measured SE (i.e., SE $\triangle \mathrm{PES}$ higher than 
zero). These L2WPMA SE approximations are marginally better than the measured SE while these SE approximations of urban OV MV BPL topologies are achieved by using higher numbers of monotonic sections.

- Suburban OV MV BPL topologies present a similar SE $\triangle$ PES behavior with urban ones. L1PMA and L2WPMA offer a marginally improved SE $\triangle$ PES behavior when high maximum values $\alpha_{\text {CUD }}$ are observed. Although the SE approximation improvement still remains marginal in the majority of the examined cases (i.e., below 1\%), a relatively high number of monotonic sections, but smaller than the number of monotonic sections used in urban OV MV BPL topologies, is required. Either urban or suburban OV MV BPL topologies are examined, L2CXCV fail in all the cases to mitigate the measurement differences. Anyway, this behavior of L2CXCV in urban and suburban OV MV BPL topologies has also been verified when L2CXCV achieves to directly mitigate the measurement differences in channel attenuations curves (see Tables 2 and 3 of [36]).

- On the basis of the achieved SE $\triangle \mathrm{PES}$, all the applied piecewise monotonic data approximations provide significant improvement concerning the mitigation of measurement differences when SE is examined. The SE $\triangle \mathrm{PES}$ improvement can reach up to $3.59 \%$ when severe measurement differences (i.e., maximum values $\alpha_{\text {CUD }}$ of $5 \mathrm{~dB}$ ) are observed. Since rural and "LOS" OV MV BPL topologies present low and rare spectral notches, simple approximations, which ignore the frequent notches imposed by the measurement differences, can achieve high SE $\triangle$ PES. Therefore, due to this poor multipath environment of rural and "LOS" OV MV BPL topologies, L1PMA and L2WPMA require low number of monotonic sections to achieve these rather simple approximations. Since the simple approximations are the more suitable for the rural and "LOS" OV MV BPL topologies, L2CXCV also achieves high $\triangle \mathrm{PES}$ in these cases.

- Although different CUDs are assumed between this paper and [36] and the nature of the measurement difference mitigation differs (i.e., direct or indirect measurement difference mitigation), the results concerning the assessment of piecewise monotonic data approximations remain approximately the same:

- The main contribution of piecewise monotonic data approximations against measurement differences is focused on rural and "LOS" OV MV BPL topologies when high maximum values $\alpha_{\text {CUD }}$ are considered. In these cases, L2WPMA and L2CXCV can offer SE $\triangle \mathrm{PES}$ that reaches up to $3.81 \%$.

- Comparing Tables 1-3 of this paper with the Tables 2-5 of [36], small differences between the relative ranking of L2WPMA and L2CXCV are due to: (i) the different CUDs that are applied in these two papers; and (ii) the nature of the measurement difference mitigation.

- With reference to the SE $\triangle \mathrm{PES}$, the best SE approximation for given OV MV BPL topology and maximum value $\alpha_{\mathrm{CUD}}=5 \mathrm{~dB}$ is highlighted with blue color in Table 2 (i.e., blue L2WPMA SE approximations).

Apart from SE $\triangle \mathrm{PES}$, to evaluate the quality of the approximations, the metrics of the set $\mathrm{A}$ and $\mathrm{B}$ of the SE approximations should tend to the respective metrics of the theoretical SE. In the following subsection, the blue L2WPMA SE approximations will 
be benchmarked against the respective theoretical SE and measured SE with reference to the metrics of set A and B.

\subsection{Case Study - Piecewise Monotonic Data Approximations and Metrics of Set $A$ and $B$}

Apart from the improvement of SE $\triangle \mathrm{PES}$, which remains the basic metric of approximation evaluation, the metrics of set A and B of SE piecewise monotonic data approximations should comply with the respective metrics of the theoretical SE so that the approximation can be considered as successful for various computations. In the case study of this subsection, the metrics of the blue L2WPMA SE approximations of Sec.4.1 will be compared against the respective ones of the theoretical and measured SE. More specifically:

- Set $A$ : The metrics of Set A are: (i) the average value of SE; (ii) the maximum value of SE; and (iii) the minimum value of SE. In Table 4, the metrics of Set A are reported for the theoretical SE, measured SE and the blue L2WPMA SE approximations for the indicative OV MV BPL topologies of this paper when the maximum value $\alpha_{\text {CUD }}$ is assumed equal to $5 \mathrm{~dB}$.

- Set B: The metrics of Set B are: (i) MAE; and (ii) RMSD. In Table 5, the metrics of Set B are reported for the theoretical SE, measured SE and the blue L2WPMA SE approximations for the indicative OV MV BPL topologies of this paper when the maximum value $\alpha_{\mathrm{CUD}}$ is assumed equal to $5 \mathrm{~dB}$.

From Tables 4 and 5, piecewise monotonic data approximations not only improve the SE subchannel estimation but also improve the macroscopic SE estimation that is described by the metrics of Set A and Set B. More specifically:

- As blue L2WPMA SE approximation is assumed, its average values of SE remains greater or equal to the respective values of measured SE in all the indicative OV MV BPL topologies that are examined. In fact, the average values of SE of the blue L2WPMA SE approximation are closer to the respective values of the theoretical SE in comparison with the respective values of SE. This is valid even if high intensity of measurement differences is considered (i.e., the maximum value $\alpha_{\mathrm{CUD}}$ is assumed equal to $5 \mathrm{~dB}$ ). Same observations can also be made in the cases of the maximum and minimum value of SE.

- MAE and RMSD describe the deviation among the approximated, measured and theoretical SE data. Hence, the metrics of set $\mathrm{B}$ again validate the mitigation success of the blue L2WPMA SE approximation against measurement differences. In all the cases examined, blue L2WPMA SE approximation achieved MAE and RMSD with values lower or equal than the respective values of the measured SE. Also, the values of MAE and RMSD of the blue L2WPMA SE approximation tend to the zero values of the theoretical SE.

- Already been mentioned in [4], the metrics of set A cannot identify the intensity of measurement differences since the differences remain marginal. In contrast, the metrics of set $\mathrm{B}$, which behave similar to PES, depend on the existence and the intensity of the measurement differences. 
Table 4. Metrics of Set A for the Theoretical SE, Measured SE and blue L2WPMA SE approximations.

\begin{tabular}{|c|c|c|c|c|c|c|c|c|c|}
\hline \multirow{3}{*}{$\begin{array}{c}\text { Indicati } \\
\text { ve OV } \\
\text { MV } \\
\text { BPL } \\
\text { Topolog } \\
\text { y }\end{array}$} & \multicolumn{9}{|c|}{ Metrics of Set A } \\
\hline & \multicolumn{3}{|c|}{$\begin{array}{c}\text { Average Value of SE } \\
(\mathrm{bps} / \mathrm{Hz})\end{array}$} & \multicolumn{3}{|c|}{$\begin{array}{c}\text { Maximum Value of SE } \\
(\mathrm{bps} / \mathrm{Hz})\end{array}$} & \multicolumn{3}{|c|}{$\begin{array}{c}\text { Minimum Value of SE } \\
(\mathrm{bps} / \mathrm{Hz})\end{array}$} \\
\hline & $\begin{array}{l}\text { Theoretic } \\
\text { al SE }\end{array}$ & $\begin{array}{c}\text { Measur } \\
\text { ed SE } \\
\left(\alpha_{\mathrm{CUD}}=5\right. \\
)\end{array}$ & $\begin{array}{c}\text { Blue } \\
\text { L2WPMA } \\
\text { SE } \\
\text { Approximati } \\
\text { on } \\
\end{array}$ & $\begin{array}{l}\text { Theoretic } \\
\text { al SE }\end{array}$ & $\begin{array}{c}\text { Measur } \\
\text { ed SE } \\
\left(\alpha_{\mathrm{CUD}}=5\right. \\
)\end{array}$ & $\begin{array}{c}\text { Blue } \\
\text { L2WPMA } \\
\text { SE } \\
\text { Approximati } \\
\text { on } \\
\end{array}$ & $\begin{array}{l}\text { Theoretic } \\
\text { al SE }\end{array}$ & 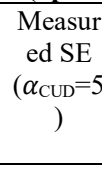 & $\begin{array}{c}\text { Blue } \\
\text { L2WPMA } \\
\text { SE } \\
\text { Approximati } \\
\text { on } \\
\end{array}$ \\
\hline $\begin{array}{r}\text { Urban } \\
\text { Case A }\end{array}$ & 10.08 & 9.86 & 9.86 & 13.60 & 14.22 & 14.22 & 1.91 & 2.36 & 2.36 \\
\hline $\begin{array}{l}\text { Suburba } \\
\text { n Case }\end{array}$ & 12.03 & 11.81 & 11.83 & 14.35 & 14.63 & 14.31 & 6.98 & 6.13 & 6.13 \\
\hline $\begin{array}{l}\text { Rural } \\
\text { Case }\end{array}$ & 13.85 & 13.60 & 13.65 & 14.25 & 14.95 & 14.34 & 12.92 & 12.32 & 12.71 \\
\hline $\begin{array}{l}\text { "LOS" } \\
\text { Case }\end{array}$ & 13.98 & 13.71 & 13.76 & 14.37 & 14.95 & 14.42 & 12.88 & 12.49 & 12.72 \\
\hline
\end{tabular}

Table 5. Metrics of Set B for the Theoretical SE, Measured SE and blue L2WPMA SE approximations.

\begin{tabular}{|c|c|c|c|c|c|c|}
\hline \multirow{2}{*}{$\begin{array}{c}\text { Indicative OV MV } \\
\text { BPL Topology }\end{array}$} & \multicolumn{4}{|c|}{$\begin{array}{c}\text { MAE } \\
\text { (bps/Hz) }\end{array}$} & \multicolumn{3}{|c|}{ Metrics of Set B } \\
\cline { 2 - 7 } & $\begin{array}{c}\text { Theoretical } \\
\text { SE }\end{array}$ & $\begin{array}{c}\text { Measured } \\
\text { SE } \\
\left(\alpha_{\text {CUD }}=5\right)\end{array}$ & $\begin{array}{c}\text { Blue } \\
\text { L2WPMA SE } \\
\text { Approximation }\end{array}$ & $\begin{array}{c}\text { Theoretical } \\
\text { SE }\end{array}$ & $\begin{array}{c}\text { Measured } \\
\text { SE } \\
\left(\alpha_{\text {CUD }}=5\right)\end{array}$ & $\begin{array}{c}\text { L2WPMA }) \\
\text { Approximation }\end{array}$ \\
\hline Urban Case A & 0 & 0.78 & 0.78 & 0 & 0.92 & 0.89 \\
\hline Suburban Case & 0 & 0.79 & 0.64 & 0 & 0.93 & 0.80 \\
\hline Rural Case & 0 & 0.76 & 0.23 & 0 & 0.89 & 0.31 \\
\hline "LOS" Case & 0 & 0.75 & 0.24 & 0 & 0.87 & 0.32 \\
\hline
\end{tabular}

- Piecewise monotonic data approximations can act as a prerequisite subsystem when measurement differences exist. In fact, their presence becomes more critical when measurement differences of high intensities are present. If the suitable piecewise monotonic data approximation is selected, the measurement difference mitigation results can be achieved regardless of the maximum value $\alpha_{\text {CUD }}$.

- The most crucial role for achieving high performances against measurement differences regardless of their maximum value $\alpha_{\text {CUD }}$ plays the selection of the suitable piecewise monotonic data approximation as well as its optimal number of monotonic sections. On the basis of the results of this Section and in accordance with [36], [37], the optimal number of monotonic sections can determine: (i) the accuracy of L1PMA and L2WPMA as expressed by PES; (ii) the accuracy of L1PMA and L2WPMA as expressed by the metrics of Set A and $\mathrm{B}$; and (iii) the performance accuracy of the piecewise monotonic data approximations that use monotonic sections against the approximations that do not use (i.e., L2CXCV).

- The indirect mitigation of measurement differences by using piecewise monotonic data approximations has revealed that even if same OV MV BPL topologies are examined the metric results are differentiated because of the different applied coupling schemes and the optimal number of monotonic sections. As concerns the direct mitigation of measurement differences by using piecewise monotonic data approximations, exhaustive investigation has been made in [33], [36], [37] for the impact of specific factors, such as the applied coupling scheme, the examined OV MV BPL topology, the maximum CUD value and the optimal number of 
monotonic sections [33], [36], [37]. The observations made there are also valid during the indirect mitigation of measurement differences.

\section{Conclusions}

This paper constitutes a case study on the indirect measurement difference mitigation during SE computations in OV MV BPL networks by applying L1PMA, L2WPMA and L2CXCV. This paper has exploited the direct measurement difference mitigation observations during channel attenuation computations in distribution BPL networks. The impact of the different piecewise monotonic data approximations, the different OV MV BPL topologies, the number of monotonic sections (when they are required) and different types and intensities of measurement differences have been assessed. With reference to PES, it has been proven that the piecewise monotonic data approximations can provide significant mitigation of measurement differences during SE computations. The SE performance characteristics of piecewise monotonic data approximations is related with the respective channel attenuation performance characteristics. Since suitable piecewise monotonic data approximations have been selected, the maximum SE, minimum SE, average SE, SE MAE and SE RMSD of the approximation present values that are closer to the theoretical ones in comparison with the respective values of the measurements. Higher measurement difference mitigation performances are achieved when higher maximum values $\alpha_{\text {CUD }}$ of the CUD measurement differences are assumed.

\section{CONFLICTS OF INTEREST}

The author declares that there is no conflict of interests regarding the publication of this paper.

\section{References}

[1] M. Emmanuel and R. Ramesh, "Evolution of Dispatchable Photovoltaic System Integration with the Electric Power Network for Smart Grid Applications: A Review," Elsevier Renewable and Sustainable Energy Reviews, vol. 67, pp. 207224, Jan. 2017.

[2] C. Zhao, J. He; P. Cheng and J. Chen, "Consensus-based Energy Management in Smart Grid with Transmission Losses and Directed Communication," IEEE Trans. on Smart Grid, vol. 8, no. 5, pp. 2049-2061, Sep. 2017.

[3] Y. Yoldaş, A. Önen, S. M. Muyeen, A. V., Vasilakos, and İ. Alan, "Enhancing Smart Grid with Microgrids: Challenges and Opportunities," Elsevier Renewable and Sustainable Energy Reviews, vol. 72, pp. 205-214, May 2017.

[4] A. G. Lazaropoulos, "Smart Energy and Spectral Efficiency (SE) of Distribution Broadband over Power Lines (BPL) Networks - Part 1: The Impact of Measurement Differences on SE Metrics," Trends in Renewable Energy, vol. 4, no. 2, pp. 125-184.

[5] A. G. Lazaropoulos, "Towards Modal Integration of Overhead and Underground Low-Voltage and Medium-Voltage Power Line Communication Channels in the Smart Grid Landscape: Model Expansion, Broadband Signal Transmission Characteristics, and Statistical Performance Metrics (Invited Paper)," ISRN Signal 
Processing, vol. 2012, Article ID 121628, pp. 1-17, 2012. [Online]. Available: http://www.hindawi.com/isrn/sp/2012/121628/

[6] A. G. Lazaropoulos, "Towards broadband over power lines systems integration: Transmission characteristics of underground low-voltage distribution power lines," Progress in Electromagnetics Research B, 39, pp. 89-114, 2012. [Online]. Available: http://www.jpier.org/PIERB/pierb39/05.12012409.pdf

[7] A. G. Lazaropoulos and P. G. Cottis, "Transmission characteristics of overhead medium voltage power line communication channels," IEEE Trans. Power Del., vol. 24, no. 3, pp. 1164-1173, Jul. 2009.

[8] A. G. Lazaropoulos and P. G. Cottis, "Capacity of overhead medium voltage power line communication channels," IEEE Trans. Power Del., vol. 25, no. 2, pp. 723-733, Apr. 2010.

[9] A. G. Lazaropoulos and P. G. Cottis, "Broadband transmission via underground medium-voltage power lines-Part I: transmission characteristics," IEEE Trans. Power Del., vol. 25, no. 4, pp. 2414-2424, Oct. 2010.

[10] A. G. Lazaropoulos and P. G. Cottis, "Broadband transmission via underground medium-voltage power lines-Part II: capacity," IEEE Trans. Power Del., vol. 25, no. 4, pp. 2425-2434, Oct. 2010.

[11] A. G. Lazaropoulos, "Broadband transmission characteristics of overhead highvoltage power line communication channels," Progress in Electromagnetics Research B, vol. 36, pp. 373-398, 2012. [Online]. Available: http://www.jpier.org/PIERB/pierb36/19.11091408.pdf

[12] A. G. Lazaropoulos, "Green Overhead and Underground Multiple-Input MultipleOutput Medium Voltage Broadband over Power Lines Networks: EnergyEfficient Power Control," Springer Journal of Global Optimization, vol. 2012 / Print ISSN 0925-5001, pp. 1-28, Oct. 2012.

[13] A. G. Lazaropoulos, "Deployment Concepts for Overhead High Voltage Broadband over Power Lines Connections with Two-Hop Repeater System: Capacity Countermeasures against Aggravated Topologies and High Noise Environments," Progress in Electromagnetics Research B, vol. 44, pp. 283-307, 2012. [Online]. Available: http://www.jpier.org/PIERB/pierb44/13.12081104.pdf

[14] A. G. Lazaropoulos, "Broadband transmission and statistical performance properties of overhead high-voltage transmission networks," Hindawi Journal of Computer Networks and Commun., 2012, article ID 875632, 2012. [Online]. Available: http://www.hindawi.com/journals/jcnc/aip/875632/

[15] P. Amirshahi and M. Kavehrad, "High-frequency characteristics of overhead multiconductor power lines for broadband communications," IEEE J. Sel. Areas Commun., vol. 24, no. 7, pp. 1292-1303, Jul. 2006.

[16] T. Calliacoudas and F. Issa, "“"Multiconductor transmission lines and cables solver," An efficient simulation tool for plc channel networks development," presented at the IEEE Int. Conf. Power Line Communications and Its Applications, Athens, Greece, Mar. 2002.

[17] T. Sartenaer and P. Delogne, "Deterministic modelling of the (Shielded) outdoor powerline channel based on the multiconductor transmission line equations," IEEE J. Sel. Areas Commun., vol. 24, no. 7, pp. 1277-1291, Jul. 2006.

[18] C. R. Paul, Analysis of Multiconductor Transmission Lines. New York: Wiley, 1994. 
[19] H. Meng, S. Chen, Y. L. Guan, C. L. Law, P. L. So, E. Gunawan, and T. T. Lie, "Modeling of transfer characteristics for the broadband power line communication channel," IEEE Trans. Power Del., vol. 19, no. 3, pp. 1057-1064, Jul. 2004.

[20] B. Li, D. Mansson, and G. Yang, "An efficient method for solving frequency responses of power-line networks," Progress In Electromagnetics Research B, Vol. 62, 303-317, 2015. doi:10.2528/PIERB15013008 http://www.jpier.org/pierb/pier.php?paper $=15013008$

[21] M. Chaaban, K. El KhamlichiDrissi, and D. Poljak, "Analytical model for electromagnetic radiation by bare-wire structures," Progress In Electromagnetics Research B, Vol. 45, 395-413, 2012. doi:10.2528/PIERB12091102 http://www.jpier.org/pierb/pier.php?paper=12091102

[22] Y. H. Kim, S. Choi, S. C. Kim, and J. H. Lee, "Capacity of OFDM two-hop relaying systems for medium-voltage power-line access networks," IEEE Trans. Power Del., vol. 27, no. 2, pp. 886-894, Apr. 2012.

[23] I. C. Demetriou and M. J. D. Powell, "Least squares smoothing of univariate data to achieve piecewise monotonicity,"IMA J. of Numerical Analysis, vol. 11, pp. 411-432, 1991.

[24] I. C. Demetriou and V. Koutoulidis, "On Signal Restoration by Piecewise Monotonic Approximation", in Lecture Notes in Engineering and Computer Science: Proceedings of The World Congress on Engineering 2013,London, U.K., Jul. 2013, pp. 268-273.

[25] I. C. Demetriou, "An application of best $L 1$ piecewise monotonic data approximation to signal restoration," IAENG International Journal of Applied Mathematics, vol. 53, no. 4, pp. 226-232, 2013.

[26] I. C. Demetriou, "L1PMA: A Fortran 77 Package for Best L1 Piecewise Monotonic Data Smoothing," Computer Physics Communications, vol. 151, no. 1, pp. 315-338, 2003.

[27] I. C. Demetriou, "Data Smoothing by Piecewise Monotonic Divided Differences,"Ph.D. Dissertation, Department of Applied Mathematics and Theoretical Physics, University of Cambridge, Cambridge, 1985.

[28] I. C. Demetriou, "Best L1 Piecewise Monotonic Data Modelling,"Int. Trans. Opl Res., vol. 1, no. 1, pp. 85-94,1994.

[29] C. de Boor, A Practical Guide to Splines. Revised Edition, NY: Springer-Verlag, Applied Mathematical Sciences, vol. 27, 2001.

[30] M. Holschneider, Wavelets. An Analysis Tool, Oxford: Clarendon Press, 1997.

[31] I. C. Demetriou, "Algorithm 863: L2WPMA, a Fortran 77 package for weighted least-squares piecewise monotonic data approximation," ACM Transactions on Mathematical Software (TOMS), vol. 33, no.1, pp. 6, 2007.

[32] I. C. Demetriou, "L2CXCV: A Fortran 77 package for least squares convex/concave data smoothing," Computer physics communications, vol. 174, no.8,pp. 643-668, 2006.

[33] A. G. Lazaropoulos, "Best L1 Piecewise Monotonic Data Approximation in Overhead and Underground Medium-Voltage and Low-Voltage Broadband over Power Lines Networks: Theoretical and Practical Transfer Function Determination," Hindawi Journal of Computational Engineering, vol. 2016, Article ID 6762390, 24 pages, 2016. doi:10.1155/2016/6762390. [Online]. Available: https://www.hindawi.com/journals/jcengi/2016/6762390/cta/ 
[34] A. G. Lazaropoulos, "Measurement Differences, Faults and Instabilities in Intelligent Energy Systems - Part 1: Identification of Overhead High-Voltage Broadband over Power Lines Network Topologies by Applying Topology Identification Methodology (TIM)," Trends in Renewable Energy, vol. 2, no. 3, pp. 85 - 112, Oct. 2016.

[35] A. G. Lazaropoulos, "Measurement Differences, Faults and Instabilities in Intelligent Energy Systems - Part 2: Fault and Instability Prediction in Overhead High-Voltage Broadband over Power Lines Networks by Applying Fault and Instability Identification Methodology (FIIM)," Trends in Renewable Energy, vol. 2, no. 3, pp. 113 - 142, Oct. 2016. [Online]. Available: http://futureenergysp.com/index.php/tre/article/view/27/33

[36] A. G. Lazaropoulos, "Power Systems Stability through Piecewise Monotonic Data Approximations - Part 1: Comparative Benchmarking of L1PMA, L2WPMA and L2CXCV in Overhead Medium-Voltage Broadband over Power Lines Networks," Trends in Renewable Energy, vol. 3, no. 1, pp. 2 - 32, Jan. 2017. [Online]. Available: http://futureenergysp.com/index.php/tre/article/view/29/34

[37] A. G. Lazaropoulos, "Power Systems Stability through Piecewise Monotonic Data Approximations - Part 2: Adaptive Number of Monotonic Sections and Performance of L1PMA, L2WPMA and L2CXCV in Overhead Medium-Voltage Broadband over Power Lines Networks," Trends in Renewable Energy, vol. 3, no. 1, pp. $33 \quad-\quad 60$, Jan. 2017. [Online]. Available: http://futureenergysp.com/index.php/tre/article/view/30/35

[38] A. G. Lazaropoulos, "Main Line Fault Localization Methodology in Smart Grid Part 1: Extended TM2 Method for the Overhead Medium-Voltage Broadband over Power Lines Networks Case," Trends in Renewable Energy, vol. 3, no. 3, pp. 2-25, Dec. $2017 . \quad$ [Online]. Available: http://futureenergysp.com/index.php/tre/article/view/36

[39] A. G. Lazaropoulos, "Main Line Fault Localization Methodology in Smart Grid Part 2: Extended TM2 Method, Measurement Differences and L1 Piecewise Monotonic Data Approximation for the Overhead Medium-Voltage Broadband over Power Lines Networks Case," Trends in Renewable Energy, vol. 3, no. 3, pp. 26-61, Dec. 2017. [Online]. Available: http://futureenergysp.com/index.php/tre/article/view/37

[40] A. G. Lazaropoulos, "Main Line Fault Localization Methodology in Smart Grid Part 3: Main Line Fault Localization Methodology (MLFLM)," Trends in Renewable Energy, vol. 3, no. 3, pp. 62-81, Dec. 2017. [Online]. Available: http://futureenergysp.com/index.php/tre/article/view/38

[41] A. G. Lazaropoulos, "Improvement of Power Systems Stability by Applying Topology Identification Methodology (TIM) and Fault and Instability Identification Methodology (FIIM) - Study of the Overhead Medium-Voltage Broadband over Power Lines (OV MV BPL) Networks Case," Trends in Renewable Energy, vol. 3, no. 2, pp. 102-128, Apr. 2017. [Online]. Available: http://futureenergysp.com/index.php/tre/article/view/34

[42] http://cpc.cs.qub.ac.uk/summaries/ADRF

[43] http://www.cpc.cs.qub.ac.uk/summaries/ADXM v1 0.html

[44] A. G. Lazaropoulos, "Factors Influencing Broadband Transmission Characteristics of Underground Low-Voltage Distribution Networks," IET Commun., vol. 6, no. 17, pp. 2886-2893, Nov. 2012. 
[45] M. Gebhardt, F. Weinmann, and K. Dostert, "Physical and regulatory constraints for communication over the power supply grid," IEEE Commun. Mag., vol. 41, no. 5, pp. 84-90, May 2003.

[46] P. S. Henry, "Interference characteristics of broadband power line communication systems using aerial medium voltage wires," IEEE Commun. Mag., vol. 43, no. 4, pp. 92-98, Apr. 2005.

[47] Ofcom, "Amperion PLT Measurements in Crieff," Ofcom, Tech. Rep., Sept. 2005.

[48] NATO, "HF Interference, Procedures and Tools (Interférences HF, procédures et outils) Final Report of NATO RTO Information Systems Technology," RTO-TRISTR-050, Jun. [Online]. 2007, Available: http://ftp.rta.nato.int/public/PubFullText/RTO/TR/RTO-TR-IST-050/\$\$TR-IST050-ALL.pdf

[49] FCC, "In the Matter of Amendment of Part 15 regarding new requirements and measurement guidelines for Access Broadband over Power Line Systems," FCC 04-245 Report and Order, Jul. 2008.

[50] Ofcom, "DS2 PLT Measurements in Crieff," Ofcom, Tech. Rep. 793 (Part 2), May 2005.

[51] Ofcom, “Ascom PLT Measurements in Winchester," Ofcom, Tech. Rep. 793 (Part 1), May 2005.

Article copyright: (C) 2018 Athanasios G. Lazaropoulos. This is an open access article distributed under the terms of the Creative Commons Attribution 4.0 International License, which permits unrestricted use and distribution provided the original author and source are credited. 